hep-th/0304115

PUPT-2081

\title{
Residues and World-Sheet Instantons
}

\author{
Chris Beasley \\ Joseph Henry Laboratories, Princeton University \\ Princeton, New Jersey 08544 \\ and \\ Edward Witten \\ School of Natural Sciences, Institute for Advanced Studies \\ Princeton, New Jersey 08540
}

\begin{abstract}
We reconsider the question of which Calabi-Yau compactifications of the heterotic string are stable under world-sheet instanton corrections to the effective space-time superpotential. For instance, compactifications described by $(0,2)$ linear sigma models are believed to be stable, suggesting a remarkable cancellation among the instanton effects in these theories. Here, we show that this cancellation follows directly from a residue theorem, whose proof relies only upon the right-moving world-sheet supersymmetries and suitable compactness properties of the $(0,2)$ linear sigma model. Our residue theorem also extends to a new class of "half-linear" sigma models. Using these half-linear models, we show that heterotic compactifications on the quintic hypersurface in $\mathbf{C P}^{4}$ for which the gauge bundle pulls back from a bundle on $\mathbf{C P}^{4}$ are stable. Finally, we apply similar ideas to compute the superpotential contributions from families of membrane instantons in M-theory compactifications on manifolds of $G_{2}$ holonomy.
\end{abstract}

April 2003 


\section{Introduction}

String theory backgrounds which preserve only $\mathcal{N}=1$ supersymmetry in four dimensions are of great interest both from a theoretical and a phenomenological perspective. A textbook way to obtain such a background is to compactify either the $E_{8} \times E_{8}$ or $\operatorname{Spin}(32) / \mathbf{Z}_{2}$ heterotic string on a Calabi-Yau threefold $X$ with a stable, holomorphic gauge bundle $E$. One might suppose that these compactifications, which admit a completely perturbative string description, would be a natural starting point from which to study the moduli space of $\mathcal{N}=1$ backgrounds of string theory.

However, in fact we know very little about which pairs $(X, E)$ give rise to consistent heterotic backgrounds, even in string perturbation theory. The issue, of course, is that models described by generic $X$ and $E$, even though they may satisfy the classical equations of motion to all orders in $\alpha^{\prime}$, are destabilized non-perturbatively by world-sheet instantons [1]. These instantons, arising from world-sheets which wrap rational (i.e. holomorphic, genus zero) curves in $X$, can each contribute to a background superpotential $W$ which lifts the Kähler moduli of $X$ and generates a cosmological constant. So one might think that the only stable $\mathcal{N}=1$ heterotic compactifications would arise from very special choices of $X$ and $E$ - for instance corresponding to world-sheet theories with $(2,2)$ supersymmetry or the $(0,2)$ models studied by Distler and Greene [2,3] - for which each world-sheet instanton simply cannot contribute to $W$.

In this light, the result of [4] that there are no non-perturbative contributions to $W$ that destabilize compactifications described by $(0,2)$ linear sigma models [5.6] is somewhat surprising. This result does not rely upon any consideration of world-sheet instantons and instead follows from simple facts about the linear sigma model. One simply observes that $W$ must always be a holomorphic section of a complex line-bundle of strictly negative curvature over the moduli space of the low-energy effective theory, which is naturally a compact Kähler manifold in the case of a linear sigma model. The compactness of the moduli space implies that $W$ must have a pole somewhere on the moduli space or else vanish identically. However, the linear sigma model, being a two-dimensional, superrenormalizable gauge theory, can only become singular when the target space becomes non-compact, as some bosonic field develops a dangerous, unsuppressed zero-mode. In computing the linear sigma model correlators which describe the couplings of gauge-singlet fields in the effective theory and so probe for a background $W$, one finds that, after suitably twisting the model, no boson has a dangerous zero-mode. So $W$ has no poles on the moduli space and thus vanishes. 
Now, Calabi-Yau compactifications which are described by $(0,2)$ linear sigma models are certainly not generic — but nor are they so special that each world-sheet instanton simply does not contribute to $W$. So from the world-sheet perspective, the stability of $(0,2)$ linear sigma models implies in these compactifications a remarkable cancellation among the contributions to $W$ from world-sheet instantons wrapped on rational curves in each homology class of $X$.

For instance, the analysis of [4] was applied in most detail to the simple case that $X$ is a quintic hypersurface in $\mathbf{C P}^{4}$ and $E$ is a deformation of the holomorphic tangent bundle $T X$, corresponding to a deformation off the locus of $(2,2)$ supersymmetric worldsheet theories. In this case, the linear sigma model result implies that, contrary to one's naive expectation, the world-sheet instanton contributions to $W$ from the 2875 lines on the generic quintic sum to zero.

Our main goal in this paper is to understand, from the world-sheet perspective, the source of this remarkable cancellation among instantons. In the process, we will introduce a new $(0,2)$ "half-linear" sigma model and show that heterotic compactifications described by these models form another class of stable $\mathcal{N}=1$ string backgrounds. For instance, using the half-linear model we show that heterotic compactifications on the quintic hypersurface in $\mathbf{C P}^{4}$ for which the gauge bundle pulls back from a bundle on $\mathbf{C P}^{4}$ are stable.

More generally, just as for the linear models, the half-linear models can be used to describe compactification on any Calabi-Yau threefold $X$ which is a complete-intersection in a compact toric variety $Y$. However, in the half-linear models the bundle $E$ on $X$ is now any stable, holomorphic bundle which pulls back from a bundle on $Y$. In particular, $E$ need not be a "monad" bundle on $X$, the sort most naturally described in the linear sigma model. Conversely, however, there are also monad bundles on $X$ (including obvious ones such as its tangent bundle) that do not pull back (at least in any obvious way) from a holomorphic bundle on $Y$. So we will also develop a version of the vanishing argument adapted to linear models and monad bundles on $X$.

1 Technically, a monad bundle is one which admits a description as the cohomology of a complex $A \rightarrow B \rightarrow C$ of three bundles $A, B$, and $C$ on $X$. 


\subsection{A Brief Sketch of the Idea}

Our essential idea can be motivated by considering the actual form of the instanton contributions to $W$ in the simple case that the string world-sheet wraps once about an isolated rational curve $C$ embedded in $X$. Actually, the most direct and elegant way [0, [8] in this case to derive the instanton contribution to $W$ is to evaluate the partition function of the worldvolume theory on a single $D 1$-brane wrapped on $C$ in the Type I theory, which is the dual description [9] of a world-sheet instanton in the $\operatorname{Spin}(32) / \mathbf{Z}_{2}$ heterotic theory 2 . Holomorphy allows us to evaluate this partition function at one-loop, so the instanton contribution to $W$ from $C$ is just

$$
W(C)=\exp \left(-\frac{A(C)}{2 \pi \alpha^{\prime}}+i \int_{C} B\right) \frac{\operatorname{Pfaff}^{\prime}\left(\mathcal{D}_{F}\right)}{\sqrt{\operatorname{det}^{\prime}\left(\mathcal{D}_{B}\right)}} .
$$

Here the exponential factor in $W(C)$ represents the classical action of the D1-brane. We have written this action in heterotic units, so that $A(C)$ is the area of $C$ in the heterotic string metric on $X, \alpha^{\prime}$ is the heterotic string tension, and $B$ is the heterotic $B$-field.

The other factor in $W(C)$ arises from the one-loop integral over the fluctuations of the bosons and fermions living on the worldvolume of the $D 1$-brane. $\mathcal{D}_{B}$ and $\mathcal{D}_{F}$ are thus the respective kinetic operators of the worldvolume bosons and fermions, and the "prime" in $\operatorname{det}^{\prime}\left(\mathcal{D}_{B}\right)$ and $\operatorname{Pfaff}^{\prime}\left(\mathcal{D}_{F}\right)$ indicates that these expressions are to be evaluated only after omitting the zero-modes associated to the bulk symmetries which are broken by the $D 1$ brane. Four bosonic zero-modes associated to the broken translational symmetries in $\mathbf{R}^{4}$ and two right-moving fermionic zero-modes associated to the broken supersymmetries arise in this fashion.

The complex structure moduli of $X$ and $E$ are described by chiral superfields in the low-energy, effective $\mathcal{N}=1$ theory, and $W(C)$ must depend holomorphically on these fields. Unfortunately, our simple expression (1.1) for $W(C)$ is not manifestly holomorphic. To get a manifestly holomorphic expression for $W(C)$, we must use the fact that the two supersymmetries left unbroken by the D1-brane imply a cancellation between the contributions of the right-moving fermionic modes to $\operatorname{Pfaff}^{\prime}\left(\mathcal{D}_{F}\right)$ and the contributions of the right-moving bosonic modes to $\operatorname{det}^{\prime}\left(\mathcal{D}_{B}\right)$.

2 As explained in [ 8$]$, the derivation of $W$ from the Type I theory most directly applies to the $\operatorname{Spin}(32) / \mathbf{Z}_{2}$ heterotic theory, but holomorphy and gauge-invariance allow us to interpret the answer for the $E_{8} \times E_{8}$ heterotic theory as well. 
To make this cancellation explicit, we write $W(C)$ solely in terms of the left-moving bosonic and fermionic modes. By convention, the kinetic operator of a left-moving fermion on $C$ will be a $\bar{\partial}$ operator, while the kinetic operator for a right-moving fermion will be a $\partial$ operator. Thus, since the left-moving worldvolume fermions transform as sections of the left-moving spin bundle $S_{-}=\mathcal{O}(-1)$ on $C$ tensored with the gauge bundle $E$ as restricted to $C$, their contribution to $\operatorname{Pfaff}^{\prime}\left(\mathcal{D}_{F}\right)$ is just the Pfaffian of the $\bar{\partial}$ operator coupled to $E \otimes \mathcal{O}(-1) \equiv E(-1)$, which we denote $\bar{\partial}_{E(-1)}$.

Similarly, in the formula (1.1) for $W(C)$, we have written the boson kinetic operator $\mathcal{D}_{B}$ as a real operator acting on the eight real bosons representing the normal directions to $C$ in $\mathbf{R}^{4} \times X$. Since $C, X$, and $\mathbf{R}^{4}$ all have complex structures, we can equally well group the eight real bosons into four complex bosons taking values in the complex normal bundle $N$ to $C$ in $\mathbf{R}^{4} \times X$. When $C$ is isolated in $X, N$ is isomorphic to $\mathcal{O} \oplus \mathcal{O} \oplus \mathcal{O}(-1) \oplus \mathcal{O}(-1)$, the first two summands representing the normal directions in $\mathbf{R}^{4}$ and the last two summands representing the normal directions in $X$. Thus, the contribution of the non-zero left-moving bosonic modes to $\operatorname{det}^{\prime}\left(\mathcal{D}_{B}\right)$ just arises from the $\bar{\partial}$ operator on $C$ coupled to the normal bundle $N$.

So, cancelling out the right-moving modes from $W(C)$ in (1.1), we have

$$
W(C)=\exp \left(-\frac{A(C)}{2 \pi \alpha^{\prime}}+i \int_{C} B\right) \frac{\operatorname{Pfaff}\left(\bar{\partial}_{E(-1)}(C)\right)}{\left(\operatorname{det}^{\prime} \bar{\partial}_{\mathcal{O}}\right)^{2}\left(\operatorname{det} \bar{\partial}_{\mathcal{O}(-1)}(C)\right)^{2}} .
$$

This expression for $W(C)$ is now manifestly holomorphic. Specifying a $\bar{\partial}$ operator on either $X$ or $E$ is equivalent to specifying its complex structure, so the operators $\bar{\partial}_{E(-1)}(C)$ and $\bar{\partial}_{\mathcal{O}(-1)}(C)$ themselves depend holomorphically on the complex structure moduli of $X$ and $E$. We have also emphasized in (1.2) that the way in which the complex structure moduli of $X$ and $E$ appear in these $\bar{\partial}$ operators itself depends upon which curve $C$ in $X$ that the instanton wraps. In fact, at least when $X$ is elliptically fibered, one can derive very explicit expressions in given examples for the dependence of $W(C)$ on the moduli of $E$ and $X$ [10,11, although we will not be needing such detailed expressions here.

Thus, in the case that $X$ is a generic quintic in $\mathbf{C P}^{4}$ and $E$ is a deformation of $T X$, the vanishing of $W$ implies as a corollary that, summing $W(C)$ over the 2875 lines on $X$,

$$
\sum_{i=1}^{2875} \frac{\exp \left(i \int_{C_{i}} B\right) \operatorname{Pfaff}\left(\bar{\partial}_{E(-1)}\left(C_{i}\right)\right)}{\left(\operatorname{det} \bar{\partial}_{\mathcal{O}(-1)}\left(C_{i}\right)\right)^{2}}=0 .
$$

3 Here $\mathcal{O}(n)$ is the usual notation for the complex line-bundle of degree $n$ on projective space. In particular, $\mathcal{O}$ is the trivial complex bundle of rank one. 
In this expression, we have dropped from $W(C)$ an overall factor of $\exp \left(\frac{-A(C)}{2 \pi \alpha^{\prime}}\right)$, which is constant for curves on $X$ of given degree, and a factor of $\left(\operatorname{det}^{\prime} \bar{\partial}_{\mathcal{O}}\right)^{-2}$, which is simply constant.

One is very much also tempted to drop from (1.3) the factor of $\exp \left(i \int_{C_{i}} B\right)$, which is at least "morally" constant on curves of given degree. However, as reviewed in [8], because only the product of $\exp \left(i \int_{C_{i}} B\right)$ and the fermion Pfaffian is even well-defined, we must technically include in (1.3) this factor involving $B$ so that the full expression makes sense. Nevertheless, our interest in (1.3) resides in the holomorphic dependence of this formula on the complex structure moduli of $X$ and $E$, and we will not dwell here on the subtleties of the heterotic $B$-field.

At first sight, the formula (1.3) might seem like an exotic mathematical prediction derived only indirectly from the underlying $(0,2)$ linear sigma model. But in fact, this sort of formula has a clear precedent from algebraic geometry, in the form of a residue theorem.

To derive the simplest example of such a residue theorem, suppose that $\omega$ is a meromorphic one-form on $\mathbf{C P}^{1}$ with simple poles at points $P_{i}, i=1, \ldots, N$. Letting $z$ be a holomorphic coordinate on $\mathbf{C P}^{1}$, we can express $\omega$ as

$$
\omega=\frac{g(z) d z}{f(z)}
$$

where $f$ and $g$ are polynomials in $z, f$ having non-degenerate zeroes at the points $P_{1}, \ldots, P_{N}$. Without loss, we assume that $\omega$ does not have a pole at $z=\infty$, so that the degrees of $f$ and $g$ satisfy

$$
\operatorname{deg} g \leq \operatorname{deg} f-2 \text {. }
$$

As usual, we then define the residue of $\omega$ at each point $P_{i}$, denoted $\operatorname{Res}_{P_{i}}(\omega)$, by integrating $\omega$ over a small contour $\gamma_{i}$ about the point $P_{i}$,

$$
\operatorname{Res}_{P_{i}}(\omega)=\frac{1}{2 \pi i} \oint_{\gamma_{i}} \omega=\frac{g\left(P_{i}\right)}{\partial f / \partial z\left(P_{i}\right)}
$$

We now obtain a residue theorem simply by considering the sum of contours

$$
\Gamma=\gamma_{1}+\cdots+\gamma_{N}
$$

Since $\Gamma$ is contractible, we have

$$
0=\frac{1}{2 \pi i} \oint_{\Gamma} \omega=\sum_{i=1}^{N} \operatorname{Res}_{P_{i}}(\omega)=\sum_{i=1}^{N} \frac{g\left(P_{i}\right)}{\partial f / \partial z\left(P_{i}\right)} .
$$


So the residue theorem simply states that the sum of the residues of $\omega$ is zero.

Comparing (1.3) to (1.8), we can already see a vague similarity between these two formulae, with the Pfaffian in (1.3) being a holomorphic function analogous to $g$ in (1.8), and the bosonic determinant in (1.3) being analogous to $\partial f / \partial z$ in (1.8). Our main goal in this paper is to make the correspondence between these formulae precise, showing directly that the instanton contributions to $W$ vanish in suitable models due to an infinite-dimensional generalization of the simple one-dimensional residue theorem above.

\subsection{The Plan of the Paper}

Our plan for the paper is as follows. In Section 2, we start by generalizing the onedimensional residue theorem to finitely many dimensions. Although standard mathematical approaches exist for studying multi-dimensional residues, as for instance in [12,13], we will take a more physical approach by studying a certain supersymmetric, finitedimensional integral. This integral is a natural abstraction of the path-integral over the right-moving world-sheet fields on the heterotic string, and from it we easily prove a very general, multi-dimensional residue theorem.

At the end of Section 2, we also describe precisely how the partition function of the worldvolume theory on a supersymmetric D1-brane can be interpreted as a residue. Unfortunately, although the D1-brane formalism provides a very elegant description of the superpotential contribution from any single instanton, the world-sheet description of the heterotic string turns out to be better for proving vanishing results for the sums of these instanton contributions.

So in Section 3, we apply our analysis from Section 2 to the heterotic world-sheet theory itself. This analysis most directly generalizes to the "half-linear" class of heterotic compactifications, for which $X$ is a complete-intersection in a compact toric variety $Y$ and the gauge bundle $E$ on $X$ pulls back from a bundle on $Y$. For these compactifications, the vanishing of the instanton contributions to $W$ follows from essentially the same argument as we use in Section 2 to deduce the multi-dimensional residue theorems. We also show how this argument can be applied to the $(0,2)$ linear sigma models to prove directly formulae such as (1.3).

Very recently, Basu and Sethi [14] have also given another argument for the stability of $(0,2)$ linear sigma models. Their argument focuses on showing the absence of corrections to the world-sheet superpotential. 
Finally, in Section 4 we consider the $\mathcal{N}=1$ compactification of M-theory on a manifold $X$ of $G_{2}$ holonomy. Using ideas very similar to those in Sections 2 and 3, we extend the results of [15] by computing the superpotential contribution from membranes which wrap a continuous family of supersymmetric three-cycles in $X$.

\section{Residues and Supersymmetry}

Rather than trying to generalize immediately from the one-dimensional residue theorem to an infinite-dimensional residue theorem which is applicable to the heterotic string, we will warm up with the simpler generalization to residue theorems in only a finite number of dimensions. Our strategy is to consider a finite-dimensional, supersymmetric integral on an arbitrary compact, complex manifold $M$. The finite-dimensional residue theorem then follows from the supersymmetry, which allows us to localize the integral to a sum of terms generalizing the one-dimensional residues, and from the compactness of $M$, which leads to the vanishing of the integral and hence the sum. After we obtain this result, we will indicate some easy generalizations of it which also have relevance to the heterotic models we introduce in Section 3. Finally, we describe precisely how the partition function of the worldvolume theory on supersymmetric $D 1$-brane can be interpreted as a residue.

Standard mathematical approaches to multi-dimensional residues and residue theorems can be found in [12] and [13]. Mathematical discussions somewhat more related to our approach via supersymmetry are given in [16], [17], and [18].

\subsection{A Finite-Dimensional Integral}

We now introduce the finite-dimensional, supersymmetric integral that is central to our study of residues and which serves as a model for the path-integral over the world-sheet fields of the heterotic string. Since the supersymmetry in our integral is essential, we will begin by specifying how it acts on the variables of integration.

As mentioned above, we perform the integral over a compact, complex manifold $M$, having (complex) dimension $n$. So the bosonic variables of integration will be the local holomorphic and anti-holomorphic coordinates $z^{i}$ and $z^{\bar{i}} \equiv \overline{z^{i}}$ on $M$.

We also introduce a set of anti-commuting, fermionic coordinates $\theta^{\bar{i}}$ and $\chi^{\alpha}$. Here the

fermions $\theta^{\bar{i}}$ transform as coordinates on the anti-holomorphic tangent bundle $\overline{T M}$, and the fermions $\chi^{\alpha}$ transform as coordinates on a holomorphic vector bundle $V$, of rank $r$, 
over $M$. The bundle $V$ is completely arbitrary and should be considered, like $M$, as part of the defining data for our integral.

Besides specifying $V$ itself, we must now also choose a global holomorphic section $s$ of $V$. We need this section $s$ simply to define an interesting supersymmetry transformation for the fermions $\chi^{\alpha}$, since none of the other variables of integration have anything to do with $V$. So under the supersymmetry $\delta$, the bosonic and fermionic variables transform as

$$
\begin{array}{ll}
\delta z^{i}=0, & \delta z^{\bar{i}}=\theta^{\bar{i}}, \\
\delta \chi^{\alpha}=s^{\alpha}, & \delta \theta^{\bar{i}}=0 .
\end{array}
$$

Note that since $s$ is holomorphic, $\delta^{2}=0$, the most important property of $\delta$.

The supersymmetric integral which we consider takes the general form

$$
Z=\int_{M} g d \mu \exp (-t S)
$$

where $t$ is a positive real parameter representing the "coupling constant" for $Z, S$ is a finite-dimensional "action" which we will soon present, and

$$
g d \mu \equiv g(z) d^{n} z d^{n} \bar{z} d^{n} \theta d^{r} \chi
$$

is the measure. Locally, $g$ is a function which represents the particular choice of measure for $Z$, and to ensure that the measure respects the supersymmetry, $g$ must be holomorphic.

The fact that we have to worry about the measure for $Z$ may seem slightly odd, since in many supersymmetric integrals, one can make a canonical choice of measure (up to normalization). The point is that, under any change of variables, the resulting Jacobians for the bosonic variables are cancelled by the fermionic Jacobians for their superpartners.

In the case of $d \mu$ above, such a cancellation occurs between the anti-holomorphic bosons $z^{\bar{i}}$ and their superpartners $\theta^{\bar{i}}$. So the factor $d^{n} \bar{z} d^{n} \theta$ appearing in $d \mu$ indeed represents a canonical choice of measure for these variables.

On the other hand, the bosonic variables $z^{i}$ and the fermionic variables $\chi^{\alpha}$ are unrelated by supersymmetry, which means that we really must choose the factor $g(z) d^{n} z d^{r} \chi$ appearing in (2.3). Globally, $g$ is not a function but transforms as a holomorphic section of the line-bundle $\Omega_{M}^{n} \otimes \wedge^{r} V$ on $M$, where $\Omega_{M}^{n}$ denotes as usual the canonical bundle of holomorphic $n$-forms on $M$ and $\wedge^{r} V$ is the top exterior power of $V$. Since we generally have no preferred choice of such a section, we must interpret our choice of $g$ as another part of the input data needed to specify $Z$. 
We must, of course, also specify the action $S$ for the integrand of $Z$. We first choose a positive-definite, hermitian metric $h_{\bar{\alpha} \alpha}$ on $V$. Then we consider a $\delta$-trivial action,

$$
S=\delta\left(h_{\bar{\alpha} \alpha} s^{\bar{\alpha}} \chi^{\alpha}\right)
$$

or expanding,

$$
S=h_{\bar{\alpha} \alpha} s^{\bar{\alpha}} s^{\alpha}+h_{\bar{\alpha} \alpha} D_{\bar{j}} s^{\bar{\alpha}} \theta^{\bar{j}} \chi^{\alpha} .
$$

Here $D_{\bar{j}}$ is the covariant derivative associated to the canonical connection arising from the metric $h_{\bar{\alpha} \alpha}$ on $V$. Recall that the canonical connection [12] is the unique connection on $V$ for which $h_{\bar{\alpha} \alpha}$ is covariantly constant and for which $D_{\bar{j}}=\partial_{\bar{j}}$ when acting on a holomorphic frame of $V$.

One easy consequence of the fact that $S$ is $\delta$-trivial is that $S$ is obviously supersymmetric, $\delta S=0$. A deeper consequence of the fact that $S$ is $\delta$-trivial is that the integral $Z$ is formally independent of the real parameter $t$ and the metric $h_{\bar{\alpha} \alpha}$ on $V$ which we introduced. For instance, the invariance of $Z$ under changes in $t$ is derived by first observing that

$$
\frac{d Z}{d t}=-\int_{M} g d \mu S \exp (-t S)=-\langle S\rangle
$$

However, if $\mathcal{O}$ is any function of the variables $z^{i}, z^{\bar{i}}, \chi^{\alpha}$, and $\theta^{\bar{i}}$, then

$$
\langle\delta \mathcal{O}\rangle=\int_{M} g d \mu \delta \mathcal{O} \exp (-t S)=0
$$

which in the language of topological field theory is the decoupling of BRST-trivial observables [19,20]. Since the action $S$ is of the form $\delta \mathcal{O}$, we deduce immediately that $d Z / d t=0$. The invariance of $Z$ under deformations of the metric $h_{\bar{\alpha} \alpha}$ follows by the same argument.

Finally, we observe that $S$ is invariant under a ghost number symmetry, under which the anti-commuting variables $\chi^{\alpha}$ and $\theta^{\bar{i}}$ carry charges -1 and +1 respectively, and $\delta$ itself carries charge +1 . Since the measure $d \mu$ thus carries ghost number

$$
\operatorname{dim} M-\operatorname{rank} V=n-r,
$$

$Z$ vanishes identically unless $n=r$. So, if we wish to use $Z$ to prove a residue theorem, we must assume that $\operatorname{dim} M=\operatorname{rank} V$. 


\subsection{A Residue Theorem}

As is familiar from the study of other topological models, we can prove an interesting theorem by using the fact that $Z$ is independent of $t$ and then evaluating $Z$ for $t \rightarrow \infty$ and $t=0$. Sometimes, a formal statement such as " $Z$ is independent of $t$ " could fail to hold if the convergence of $Z$ were sufficiently poor. See [4] for a nice demonstration of such a failure in the context of the linear sigma model. However, because here $Z$ is an integral over a compact manifold $M$, the convergence of $Z$ is assured, even when $t=0$, and there are no difficulties with the formal statements above.

Evaluating $Z$ when $t=0$ is easy. Then

$$
Z=\int_{M} g d \mu 1=0
$$

since neither $\chi^{\alpha}$ nor $\theta^{\bar{i}}$ appear in the integrand above.

Evaluating $Z$ for $t \rightarrow \infty$, we see from the action $S$ in (2.5) that only points in a neighborhood of the vanishing locus $L$ of the section $s$ contribute to $Z$. In general, $L$ will consist of several disconnected components $C$, and $Z$ must have an expression

$$
Z=\sum_{C \subset L} Z(C)
$$

where $Z(C)$ denotes the local contribution to $Z$ from the component $C$. So (2.9) and (2.10) imply as a very general vanishing theorem that

$$
\sum_{C \subset L} Z(C)=0
$$

The power of this approach is that the vanishing theorem (2.11) does not rely on any specific behavior of the section $s$ of $V$. In the simplest case, $s$ has simple zeroes on a set of isolated points of $M$. But we can equally well consider the case that $s$ has degenerate zeroes at some points, or even that $s$ vanishes over some components of positive dimension. In order to translate (2.11) into a more explicit formula, along the lines of the one-dimensional residue theorem (1.8), we must simply evaluate the expression $Z(C)$ for each case.

\section{Multi-dimensional residues}

To make contact with the one-dimensional residue theorem (1.8), we will consider at first only the easiest case that $s$ vanishes in a non-degenerate fasion on a set of isolated points $P$ of $M$. 
Recall that the requirement that $s$ vanish non-degenerately at a point $P$ is simply the condition that the Jacobian $\operatorname{det}(d s)$ be non-vanishing at $P$,

$$
\operatorname{det}(d s)(P)=\operatorname{det}\left(\frac{\partial\left(s^{1}, \cdots, s^{n}\right)}{\partial\left(z^{1}, \cdots, z^{n}\right)}\right)(P) \neq 0
$$

In this case, the contribution $Z(P)$ from $P$ can be evaluated exactly using the Gaussian approximation to $Z$ near this point, and we easily see that

$$
Z(P)=\frac{g(P)}{\operatorname{det}(d s)(P)}
$$

Thus, the vanishing result (2.11) becomes

$$
\sum_{P \in L} \frac{g(P)}{\operatorname{det}(d s)(P)}=0
$$

This expression represents a natural generalization of the one-dimensional residue theorem $(1.8)$.

To sharpen the correspondence between the formula (2.14) and a multi-dimensional residue theorem, we consider the particular case that the bundle $V$ is a direct sum of $n$ line bundles,

$$
V=\mathcal{O}\left(D_{1}\right) \oplus \cdots \oplus \mathcal{O}\left(D_{n}\right)
$$

which are associated to $n$ irreducible, effective divisors $D_{1}, \ldots, D_{n}$ intersecting transversely at isolated points $P$ in $M$.

To describe the appropriate section $s$ of $V$ for this case, we note that each divisor $D_{i}$ is determined as the vanishing locus of a holomorphic section $s_{i}$ of the line-bundle $\mathcal{O}\left(D_{i}\right)$. Then we simply take $s$ to be the direct sum of the $s_{i}$, so that $s$ has components

$$
s=\left(s_{1}, \ldots, s_{n}\right) .
$$

We note that the section $s$ vanishes non-degenerately at each point $P \in D_{1} \cap \cdots \cap D_{n}$, so our simple expression for $Z(P)$ in (2.13) is valid.

In this case, we can now give a very nice geometric interpretation of the local contribution $Z(P)$ from each point $P \in D_{1} \cap \cdots \cap D_{n}$. Near $P$, we can trivialize all the line bundles $\mathcal{O}\left(D_{i}\right)$ as well as the canonical bundle of $M$. Upon doing so, we can regard

4 In the following, we suppress overall factors of $\pi$ that arise from the Gaussian integration. 
$g$ as an ordinary holomorphic function that is nonzero at $P$, and the $s_{i}$ as holomorphic functions that vanish on $D_{i}$. Now we can define a meromorphic $n$-form $\omega$ that generalizes the one-dimensional expression (1.4),

$$
\omega=\frac{g d z^{1} \wedge \cdots \wedge d z^{n}}{s_{1} \cdots s_{n}}
$$

Given the meromorphic $n$-form $\omega$, and a real $n$-cycle $\gamma$ that links in a suitable way the locus of its poles, we can naturally define an $n$-dimensional residue $\operatorname{Res}_{P}(\omega)=$ $(1 / 2 \pi i)^{n} \int_{\gamma} \omega$ that will generalize the usual one-dimensional residue. We let $\gamma$ be the real $n$-cycle determined by

$$
\left|s_{i}\right|=\epsilon, \quad i=1, \ldots, n
$$

where $\epsilon$ is a small parameter. Technically, we must also orient $\gamma$, which we do by the condition $d\left(\arg s_{1}\right) \wedge \cdots \wedge d\left(\arg s_{n}\right) \geq 0$.

On $\gamma, \omega$ is holomorphic, so we can define

$$
\operatorname{Res}_{P}(\omega)=\left(\frac{1}{2 \pi i}\right)^{n} \int_{\gamma} \omega .
$$

Since $d \omega=0$ on a neighborhood of $\gamma$, this definition only depends on the homology class of $\gamma$ and in particular does not depend the parameter $\epsilon$ above.

The residue $\operatorname{Res}_{P}(\omega)$ can be then be evaluated by a change of variables and the iterative application of Cauchy's theorem. We find

$$
\operatorname{Res}_{P}(\omega)=\frac{g(P)}{\operatorname{det}(d s)(P)},
$$

generalizing the one-dimensional expression in (1.6). Of course, $\operatorname{Res}_{P}(\omega)$ agrees precisely with $Z(P)$ for the special choices of $V$ and $s$ above, so our main result (2.11) is properly interpreted as a generalized, multi-dimensional residue theorem.

\section{A quick example}

Before proceeding further, we will give a quick example of the residue theorem.

For our example, we take $M=\mathbf{C P}^{2}$ and $V=T M$, the holomorphic tangent bundle. If we let $\left[X_{0}: X_{1}: X_{2}\right]$ be homogeneous coordinates on $M$, then any holomorphic section $s$ of $V$ takes the form

$$
s=a_{0} X_{0} \frac{\partial}{\partial X_{0}}+a_{1} X_{1} \frac{\partial}{\partial X_{1}}+a_{2} X_{2} \frac{\partial}{\partial X_{2}},
$$


where $\left(a_{0}, a_{1}, a_{2}\right)$ are complex coefficients parametrizing $s$. Because $\left[X_{0}: X_{1}: X_{2}\right]$ are only homogeneous coordinates, the coefficients $\left(a_{0}, a_{1}, a_{2}\right)$ are only defined up to the addition of a multiple of $(1,1,1)$, which describes the zero section of $V$. If $\left(a_{0}, a_{1}, a_{2}\right)$ are generic coefficients, then $s$ vanishes non-degenerately at the three points $P_{1}=[1: 0: 0]$, $P_{2}=[0: 1: 0]$, and $P_{3}=[0: 0: 1]$ of $M$.

Since $V=T M$, the measure $d \mu$ is a section of the trivial bundle $\mathcal{O}=\Omega_{M}^{n} \otimes \wedge^{n} T M$. Consequently, in this example we do have a canonical measure for $Z$ and $g$ is a constant.

Now in the patch where $X_{0} \neq 0$, with local coordinates $\left(z^{1}, z^{2}\right), s$ takes the form

$$
s=\left(a_{1}-a_{0}\right) z^{1} \frac{\partial}{\partial z^{1}}+\left(a_{2}-a_{0}\right) z^{2} \frac{\partial}{\partial z^{2}}
$$

and so the residual contribution from $P_{1}$ to $Z$ is

$$
Z\left(P_{1}\right)=\frac{1}{\left(a_{1}-a_{0}\right)\left(a_{2}-a_{0}\right)}
$$

Similar contributions from the points $P_{2}$ and $P_{3}$ are

$$
Z\left(P_{2}\right)=\frac{1}{\left(a_{0}-a_{1}\right)\left(a_{2}-a_{1}\right)}, \quad Z\left(P_{3}\right)=\frac{1}{\left(a_{0}-a_{2}\right)\left(a_{1}-a_{2}\right)} .
$$

The residue theorem then simply states that $Z\left(P_{1}\right)+Z\left(P_{2}\right)+Z\left(P_{3}\right)=0$, as one can verify directly.

\subsection{Generalizations}

The ghost number symmetry preserved by $S$ implies that $Z$ trivially vanishes unless $\operatorname{rank} V=\operatorname{dim} M$. So if we wish to study bundles $V$ such that $\operatorname{rank} V \neq \operatorname{dim} M$, we should consider not $Z$ itself but expectation values $\langle\mathcal{O}\rangle$,

$$
\langle\mathcal{O}\rangle=\int_{M} g d \mu \mathcal{O} \exp (-t S)
$$

where $\mathcal{O}$ is any function of $z^{i}, z^{\bar{i}}, \chi^{\alpha}$, and $\theta^{\bar{i}}$ which satisfies $\delta \mathcal{O}=0$. Of course, $\mathcal{O}$ must also have ghost number $n-r$ if $\langle\mathcal{O}\rangle$ is to be any more interesting that $Z$ itself.

Globally, functions $\mathcal{O}$ of $z^{i}, z^{\bar{i}}, \chi^{\alpha}$, and $\theta^{\bar{i}}$ are elements of the complex

$$
\bigoplus_{(p, q)} A^{(0, q)}(M) \otimes \wedge^{p} V^{*}
$$


Here $A^{(0, q)}(M)$ is the bundle of smooth $(0, q)$ forms on $M$, and $V^{*}$ is the holomorphic bundle dual to $V$. A function homogeneous and $q^{t h}$ order in $\theta^{\bar{i}}$ is a $(0, q)$-form on $M$, while a function homogeneous and $p^{\text {th }}$ order in $\chi^{\alpha}$ is a section of $\wedge^{p} V^{*}$. We will often refer to an element of $A^{(0, q)}(M) \otimes \wedge^{p} V^{*}$ for fixed $(p, q)$ as having "type" $(p, q)$.

The supersymmetry transformation $\delta$ acts on elements of this complex as

$$
D=\theta^{\bar{i}} \frac{\partial}{\partial z^{\bar{i}}}+s^{\alpha} \frac{\partial}{\partial \chi^{\alpha}} .
$$

More intrinsically, we can identify $D$ with the operator

$$
D=\bar{\partial}+\iota_{s},
$$

where $\bar{\partial}$ is the usual Dolbeault operator on $M$ and $\iota_{s}$ acts on sections of $\wedge^{p} V^{*}$ by the interior product with $s$. The action of $D$ on this complex has certainly been considered before in the mathematical literature, for instance in [16, 17, 18, , though mostly for the case $V=T M$.

Since $\langle\delta \mathcal{O}\rangle=0$ for any $\mathcal{O}$, the interesting observables $\mathcal{O}$ correspond to nontrivial elements of the cohomology of $D$. In general, what can we say about this cohomology?

Without placing additional conditions on $M, V$, and $s$, in fact we cannot say much Nonetheless, we do have a systematic procedure to compute the $D$-cohomology, using a spectral sequence (see [21] for a clear introduction to spectral sequences).

In physical terms, we want to solve the equation $\delta \mathcal{O}=0$, and the spectral sequence is essentially a perturbative way to do this, really by following one's nose. So to construct an $\mathcal{O}$ which satisfies $\delta \mathcal{O}=0$, we start with an "order-zero" trial solution $\mathcal{O}^{(0)}$, of type $(p, q)$, which satisfies $6 \bar{\partial} \mathcal{O}^{(0)}=0$. If $\mathcal{O}^{(0)}$ also happens to satisfy $\iota_{s} \mathcal{O}^{(0)}=0$, then $\mathcal{O}=\mathcal{O}^{(0)}$, but generally $\iota_{s} \mathcal{O}^{(0)} \neq 0$.

To correct for this discrepancy, we then try to solve

$$
\iota_{s} \mathcal{O}^{(0)}+\bar{\partial} \mathcal{O}^{(1)}=0
$$

${ }^{5}$ However, see [18 for a nice discussion of the easiest case that $V=T M$ and $s$ has zeroes at isolated points. In this case, the cohomology of $D$ is isomorphic to $H^{0}(M, \mathcal{O} / \mathcal{I})$, where $\mathcal{I}$ is the ideal sheaf associated to $s$.

6 If we wished, we could equally well start with $\mathcal{O}^{(0)}$ satsifying $\iota_{s} \mathcal{O}^{(0)}=0$ and reverse the roles of $\bar{\partial}$ and $\iota_{s}$ above. We find it convenient to do this in Section 3 . 
to determine the "first-order" correction $\mathcal{O}^{(1)}$. We consider $\mathcal{O}^{(1)}$ as a correction to $\mathcal{O}^{(0)}$ in a very definite sense, since although $\mathcal{O}^{(0)}$ is of type $(p, q), \mathcal{O}^{(1)}$ is of type $(p-1, q-1)$. Thus, if we continue to solve iteratively

$$
\iota_{s} \mathcal{O}^{(n)}+\bar{\partial} \mathcal{O}^{(n+1)}=0,
$$

we will either find an obstruction, or the procedure will terminate after a finite number of steps with $\mathcal{O}=\mathcal{O}^{(0)}+\mathcal{O}^{(1)}+\cdots$ satisfying $\delta \mathcal{O}=0$. We will find this little procedure useful when constructing heterotic models in Section 3.

What sort of results, analogous to the generalized residue theorem (2.11), do we then obtain by considering the expectations $\langle\mathcal{O}\rangle$ of nontrivial observables $\mathcal{O}$ ? Evaluating $\langle\mathcal{O}\rangle$ at $t=0$ now yields

$$
\langle\mathcal{O}\rangle=\int_{M} g d \mu \mathcal{O},
$$

which need not vanish if $\mathcal{O}$ carries the proper ghost number. Evaluating $\langle\mathcal{O}\rangle$ in the limit $t \rightarrow \infty$, we again see that $\langle\mathcal{O}\rangle$ can be expressed as a sum of local contributions from each of the components $C$ of the vanishing locus $L$ of $s$,

$$
\langle\mathcal{O}\rangle=\sum_{C \subset L}\langle\mathcal{O}\rangle(C) .
$$

So for instance, again in the case that $s$ vanishes non-degenerately over isolated points $P$ of $M$ and $\mathcal{O}$ has ghost number zero,

$$
\int_{M} g d \mu \mathcal{O}=\sum_{P \in L} \frac{g(P) \mathcal{O}(P)}{\operatorname{det}(d s)(P)} .
$$

In the above expression, we must interpret the integral over $M$ as picking out the component of $\mathcal{O}$ of type $(n, n)$ and the evaluation at $P$ as picking out the component of $\mathcal{O}$ of type $(0,0)$.

We can also consider the under-determined case, for which $\operatorname{rank} V<\operatorname{dim} M$, as well as the over-determined case, for which $\operatorname{rank} V>\operatorname{dim} M$. In the under-determined case, the components $C$ of $L$ will generically be complex submanifolds of dimension $n-r$ in $M$. We assume that $s$ vanishes in a non-degenerate fashion on each $C$, which means that the Jacobian $\operatorname{det}\left(\left.d s\right|_{N}\right)$ of $s$ with respect to the normal directions to $C$ in $M$ is non-vanishing along $C$. Then the local contribution of $C$ to $\langle\mathcal{O}\rangle$ is

$$
\langle\mathcal{O}\rangle(C)=\int_{C} \frac{g d \mu \mathcal{O}}{\operatorname{det}\left(\left.d s\right|_{N}\right)} .
$$


In the above, $g d \mu / \operatorname{det}\left(\left.d s\right|_{N}\right)$ determines an element of $\Omega^{n-r}$ on $C$, and thus only the component of $\mathcal{O}$ of type $(0, n-r)$ now contributes to the integral over $C$.

As we shall see in Section 3, the case of direct relevance to the heterotic string is actually the over-determined case, $\operatorname{rank} V>\operatorname{dim} M$. In this case, one might think that it is unnatural to consider an $s$ that has zeroes. However, to get a non-trivial result in the over-determined case, we need a non-trivial $\mathcal{O}$, most simply an $\mathcal{O}$ of degree $(r-n, 0)$. In any case, such an $\mathcal{O}$ will be present when we study the half-twisted heterotic string. $(r-n$ will be infinite, and $\mathcal{O}$ will be the exponential of a fermion bilinear.) When such an $\mathcal{O}$ is present, $s$ cannot be changed freely, since one must preserve the condition $\left(\bar{\partial}+\iota_{s}\right) \mathcal{O}=0$. In the presence of a suitable $\mathcal{O}$, it can be natural to have an $s$ that has zeroes with nontrivial residues. For instance, if $s$ again vanishes non-degenerately at an isolated point $P$ of $M$, now meaning that the matrix $d s=\left(\partial s^{\alpha} / \partial z^{i}\right)$ has full rank at $P$, then the local contribution from $P$ to $\langle\mathcal{O}\rangle$ is

$$
\langle\mathcal{O}\rangle(P)=\left(\frac{g d \mu \mathcal{O}}{d s}\right)(P) \equiv\left(\frac{g \epsilon_{i_{1} \cdots i_{q}} \epsilon^{\alpha_{1} \cdots \alpha_{p}} \mathcal{O}_{\alpha_{q+1} \cdots \alpha_{p}}}{\partial_{i_{1}} s^{\alpha_{1} \cdots \partial_{i_{q}} s^{\alpha_{q}}}}\right)(P)
$$

Evidently, in such an example with isolated zeroes of $s$, only the component of type $(r-n, 0)$ of $\mathcal{O}$ contributes to $\langle\mathcal{O}\rangle(P)$.

\subsection{The D1-brane Partition Function as a Residue}

Our discussion of multi-dimensional residues now allows us to make precise the manner in which the partition function of a supersymmetric D1-brane can be interpreted as a residue. We have already seen in the Introduction a strong formal similarity between expressions such as (1.3) and (1.8) which suggests this interpretation. To check this idea, though, we must examine to what extent the worldvolume theory on a supersymmetric D1-brane actually generalizes our finite-dimensional model which produces the residues.

At first glance, one might be worried by the following fact. If we consider the bosonic action for a $D 1$-brane which wraps an arbitrary, not necessarily holomorphic, surface $\Sigma$ in the Calabi-Yau threefold $X$, then this action is just the area $A[\Sigma]$ of the surface. So if the D1-brane action were literally to be the obvious generalization of the action (2.5) of the finite-dimensional model, then $A[\Sigma]$ would have to admit a representation as the norm-squared of a suitable holomorphic section $s$ over the space $\mathcal{M}$ of immersed surfaces in $X$. But $A[\Sigma]$ presumably does not admit such a representation, and it is not even 
obvious that the space $\mathcal{M}$, which should play the role of the complex manifold $M$ in the finite-dimensional model, admits a complex structure.

Thus, as far as we know, the full D1-brane worldvolume action does not fit into the simple structure of the finite-dimensional model. As a result, we cannot hope to use the $D 1$ brane formalism to prove vanishing results such as (1.3). Physically, the difficulty in using the $D 1$-brane formalism to prove the vanishing results is that the $D 1$-brane worldvolume description becomes more complicated when the brane is "off-shell", i.e. not supersymmetric. We do not believe that these off-shell complications are really essential, but we also do not know how to eliminate them in the D1-brane framework 0 When we deduce these vanishing results in Section 3, we will use instead approaches based on linear and half-linear sigma models, which are more closely related to the finite-dimensional model.

Yet to discuss the superpotential contribution from a D1-brane which wraps an isolated holomorphic curve $C$ in $X$ requires considerably less than the full worldvolume action. Since we evaluate the partition function at one-loop, we only need to discuss fluctuations about the holomorphic curve up to quadratic order in the action. Considering the worldvolume theory only to this order, we can nicely fit it into the framework of the finitedimensional model. In particular, the second variation of $A[\Sigma]$ away from a minimum corresponding to a holomorphic curve indeed appears as the norm-squared of a suitable section $s$ and the contribution to the superpotential is indeed a residue.

Geometrically, the approach of working only to quadratic order in the supersymmetric D1-brane action corresponds to linearizing the space $\mathcal{M}$ over the point corresponding to the given holomorphic curve $C$ in $X$. The linearization possesses the requisite complex structure.

We now give a thorough discussion of how this approximation to the D1-brane action fits into the framework of the finite-dimensional model. As we have indicated, our identification of the supersymmetric D1-brane partition function as a residue is of more conceptual than practical interest here, not only because of the off-shell complications but also because of the lack of compactness in the D1-brane approach. However, in Section 4 we will apply similar ideas to study the superpotential contributions from continuous families of membrane instantons in M-theory compactifications on manifolds of $G_{2}$ holonomy.

To proceed, we begin with the general observation [22] that whenever a brane wraps a supersymmetric cycle, then the worldvolume theory on the brane is automatically twisted,

7 D5-branes can be put in a gauge-invariant version of this framework. 
implying the existence of at least one scalar supercharge. The existence of a scalar supercharge on the D1-brane worldvolume is crucial if we are to interpret the worldvolume theory in analogy to the finite-dimensional model, with its scalar supersymmetry generator $\delta$.

We focus our attention on the sector of the D1-brane worldvolume theory describing fluctuations of the brane in $X$, as opposed to the trivial sector describing fluctuations in $\mathbf{R}^{4}$. When the $D 1$-brane wraps a holomorphic curve $C$ in $X$, the worldvolume bosons $x^{i}$ and $x^{\bar{i}}$ which describe fluctuations of the brane in $X$ transform as coordinates on the holomorphic normal bundle $N$ and anti-holomorphic normal bundle $\bar{N}$ of $C$ in $X$. The worldvolume theory also possesses fermions $\psi_{\dot{\alpha}, i}$ which transform as right-moving Weyl fermions on $\mathbf{R}^{4}$, as indicated by the $\dot{\alpha}$ index, and as coordinates on the dual bundle $N^{*}$ of $N$. Equivalently, using the hermitian metric $g_{\bar{i} i}$ on $X$, we can regard these fermions as transforming in the anti-holomorphic normal bundle $\bar{N}$. The twisted model has two scalar supercharges, described in detail later, which relate the worldvolume fields $\left(x^{\bar{i}}, \psi_{\dot{\alpha}}^{\bar{i}}\right)$.

Now, in the finite-dimensional model, the supersymmetry transformations as well as the form of the action are determined by the holomorphic section $s$ of $V$. So what are the analogues of $s$ and $V$ for the $D 1$-brane?

As has already been observed in [23,24, for a variety of supersymmetric compactifications of string and M-theory, the supersymmetric brane configurations can be characterized as the critical points of a "superpotential" $\Psi$, suitably interpreted as a function on the space of arbitrary brane configurations. (This idea has also been discussed lately in a mathematical context in [25].) For the D1-brane, if $\delta$ is the exterior derivative on the space $\mathcal{M}$ of brane configurations, then $\delta \Psi$ is a one-form that vanishes at the point corresponding to a holomorphic curve $C$, and moreover $\delta \Psi$ is holomorphic once we linearize in a neighborhood of $C$. So a natural guess is to take $V$ to be the holomorphic cotangent bundle $T^{*} \mathcal{M}$ and $s=\delta \Psi$.

To check that this identification is correct, we must describe $\Psi$ explicitly. For argument's sake, we start by defining $\Psi$ on surfaces $\Sigma$ which are homologically trivial in $X$ - although we note that any holomorphic surface, being calibrated by the Kähler form on $X$, actually resides in a nontrivial homology class. In any case, $\Psi(\Sigma)$ is defined for a homologically trivial surface $\Sigma$ by

$$
\Psi(\Sigma)=\frac{1}{6} \int_{B} \Omega
$$


where $B$ is a bounding three-cycle for $\Sigma$ and $\Omega$ is the holomorphic three-form on $X$. The factor of $\frac{1}{6}$ is simply to cancel some constants that would otherwise appear in later formulae. If $H_{3}(X, \mathbf{Z}) \neq 0$, as is always the case when $X$ has complex structure moduli, then $\Psi(\Sigma)$ generally depends on the class of $B$ and is defined only up to an additive constant.

Now, if $\Sigma$ is a surface representing a nontrivial homology class in $X$, then a bounding three-cycle $B$ does not exist. To define $\Psi(\Sigma)$ in this case, for each class in $H_{2}(X, \mathbf{Z})$ we choose a particular representative $\Sigma_{0}$. Then, if $\Sigma$ lies in the same class as $\Sigma_{0}$, a bounding three-cycle $B$ exists for $\Sigma-\Sigma_{0}$. That is, the boundary of $B$ has two components, one of which is $\Sigma$ and the other is $\Sigma_{0}$, considered with opposite orientation. So now we set

$$
\Psi(\Sigma)-\Psi\left(\Sigma_{0}\right)=\frac{1}{6} \int_{B} \Omega .
$$

In this case, the additive constant in $\Psi$ also depends on the representative $\Sigma_{0}$ as well as the class of $B$.

The fact that $\Psi$ is only defined up to an additive constant does not concern us, as this constant does not affect the location of the critical points, for which $\delta \Psi=0$. Explicitly, in terms of holomorphic coordinates $x^{i}$ on $X$,

$$
\delta \Psi(\Sigma)=\frac{1}{2} \int_{\Sigma} \Omega_{i j k} \delta x^{i} d x^{j} \wedge d x^{k} .
$$

So $\delta \Psi=0$ precisely for those surfaces $\Sigma$ on which the $(2,0)$-form $\Omega_{i j k} d x^{j} \wedge d x^{k}$ is equal to zero. If $\Sigma$ is holomorphic in $X$, then any $(2,0)$-form vanishes when restricted to $\Sigma$, so $\delta \Psi$ vanishes when $\Sigma$ is a holomorphic curve $C$. Because $\Omega_{i j k}$ is everywhere nonzero, holomorphy of $\Sigma$ is necessary as well as sufficient for vanishing of $\delta \Psi$.

While $\delta \Psi$ vanishes at the point corresponding to $C$, we also need the linear behavior near this point. For this, we pick local complex coordinates on $X$ consisting of a parameter $z$ that is a local complex coordinate on $C$ as well as two local coordinates $y^{i}$ of the normal bundle $N$. We write $\epsilon_{i j}$ for $\Omega_{z i j}$. In (2.38), we take $\delta x^{i}$ to be a displacement of one of the $y^{i}$, since otherwise we are not moving $\Sigma$ away from $C$ at all. So we will write $\delta y^{i}$ for $\delta x^{i}$. Evaluated on $\Sigma$, we have $d x^{j} \wedge d x^{k}=d z \wedge d \bar{z}\left(\partial_{z} x^{j} \partial_{\bar{z}} x^{k}-\partial_{\bar{z}} x^{j} \partial_{z} x^{k}\right)$. Because of the antisymmetry in $j$ and $k$ (or because $\partial_{\bar{z}} z=0$ ), we cannot set both $x^{j}$ and $x^{k}$ equal to $z$. To linearize $\delta \Psi$ around $C$, we set one of them, say $x^{j}$, to $z$, and the other to $y^{k}$. So we get

$$
\delta \Psi=\int_{C} \epsilon_{i j} \delta y^{i} \bar{\partial} y^{j}+\ldots
$$


where the ellipses indicate that higher order terms have been dropped. From this, we can also deduce that to quadratic order,

$$
\Psi=c+\frac{1}{2} \int_{C} \epsilon_{i j} y^{i} \bar{\partial} y^{j},
$$

where $c$ is an integration constant.

In particular, we see from (2.40) that when evaluated on $C$,

$$
\left.\frac{\delta^{2} \Psi}{\delta y^{j}(z) \delta y^{i}\left(z^{\prime}\right)}\right|_{C}=\epsilon_{i j} \partial_{\bar{z}} \delta\left(z, z^{\prime}\right),
$$

where, more intrinsically, $\partial_{\bar{z}}$ represents the $\bar{\partial}$ operator acting on sections of $N$.

Since $\Psi$ functions like a superpotential, the unbroken worldvolume supersymmetries in the linearized theory can be very simply expressed in terms of $\Psi$. Under the twisted supercharges $\bar{Q}_{\dot{\alpha}}$, the transformations of the fields $y^{i}, y^{\bar{i}}$, and $\psi_{\dot{\alpha}, i}$ take the usual form

$$
\begin{aligned}
& \delta_{\dot{\alpha}} y^{i}=0, \quad \delta_{\dot{\alpha}} y^{\bar{i}}=\psi_{\dot{\alpha}}^{\bar{i}}, \\
& \delta_{\dot{\alpha}} \psi_{\dot{\beta}, i}=\epsilon_{\dot{\alpha} \dot{\beta}} \frac{\delta \Psi}{\delta y^{i}} .
\end{aligned}
$$

Since $\Psi$ is holomorphic, in the sense that $\delta \Psi / \delta y^{\bar{i}}=0$, these supersymmetry transformations satisfy $\left\{\delta_{\dot{\alpha}}, \delta_{\dot{\beta}}\right\}=0$ as required. Obviously these worldvolume supersymmetries are unbroken when $\delta \Psi / \delta y^{i}=0$, which we have already observed is the proper condition for the $D 1$-brane to be supersymmetric. Further, taking $s=\delta \Psi$, we see that (2.42) represents an $N=2$ generalization of the supersymmetry transformations (2.1) in the finite-dimensional model.

The worldvolume action which describes to leading order the fluctuations of a $D 1$ brane which wraps a holomorphic curve $C$ in $X$ takes a very simple form when written in terms of $\Psi$. Just as for the finite-dimensional action (2.4),

$$
\begin{aligned}
S & =\frac{1}{4} \int_{C} \epsilon^{\dot{\alpha} \dot{\beta}} \delta_{\dot{\beta}}\left(\omega g^{\bar{i} i} \frac{\delta \bar{\Psi}}{\delta y^{\bar{i}}} \psi_{\dot{\alpha}, i}\right) \\
& =\int_{C} \omega\left(\frac{1}{2} g^{\bar{i} i} \frac{\delta \bar{\Psi}}{\delta y^{\bar{i}}} \frac{\delta \Psi}{\delta y^{i}}+\frac{1}{4} \epsilon^{\dot{\alpha} \dot{\beta}} g^{\bar{i} i} \frac{D^{2} \bar{\Psi}}{D y^{\bar{j}} D y^{\bar{i}}} \psi_{\dot{\beta}}^{\bar{j}} \psi_{\dot{\alpha}, i}\right) .
\end{aligned}
$$

Here $D$ is the covariant derivative with respect to the metric $g_{i \bar{i}}$ on $X$, and $\omega$ is the Kähler form on $X$ which restricts to the volume form on $C$. We also note from (2.38) that $\delta \Psi / \delta y^{i}$ is actually a two-form on $C$, and we have implicitly used the induced metric to dualize $\delta \Psi / \delta y^{i}$ to a scalar on $C$. 
The action $S$ is to be interpreted by expanding to quadratic order in the normal fluctuations $y^{i}$ and $y^{\bar{i}}$ about the given holomorphic curve $C$, so that

$$
S=\int_{C} \omega\left(\frac{1}{2} g^{\bar{i} i} \frac{D^{2} \bar{\Psi}}{D y^{\bar{j}} D y^{\bar{i}}} \frac{D^{2} \Psi}{D y^{i} D y^{j}} y^{\bar{j}} y^{j}+\frac{1}{4} \epsilon^{\dot{\alpha} \dot{\beta}} g^{\bar{i} i} \frac{D^{2} \bar{\Psi}}{D y^{\bar{j}} D y^{\bar{i}}} \psi_{\dot{\beta}}^{\bar{j}} \psi_{\dot{\alpha}, i}\right) .
$$

Using (2.41), we can write $S$ more explicitly as

$$
S=\frac{1}{2} \int_{C} \omega\left(g_{\bar{i} i} \partial_{z} y^{\bar{i}} \partial_{\bar{z}} y^{i}+\frac{1}{2} \epsilon^{\dot{\alpha} \dot{\beta}} \epsilon_{\overline{i j}} \psi_{\dot{\beta}}^{\bar{j}} \partial_{z} \psi_{\dot{\alpha}}^{\bar{i}}\right)
$$

In the above, we have assumed that $\Omega$ is normalized on $X$ so that $g^{\bar{j} j} \bar{\epsilon}_{\overline{j i}} \epsilon_{j i}=g_{\bar{i} i}$. This action is just the free action for fluctuations which we implicitly used in the Introduction when we evaluated the partition function.

More geometrically, we can identify the complex linear space $\mathcal{M}$ describing fluctuations of the $D 1$-brane about $C$ with the space of sections of $N$. Our formula for $S$ simply reflects the classic fact [26,27] that, given a section $y^{i}$, the second derivative of the area functional $A\left[\Sigma_{t}\right]$ along the one-parameter family of surfaces $\Sigma_{t}$ determined by $y^{i}$, evaluated at $\Sigma_{0}=C$, is just

$$
\left.\frac{d^{2}}{d t^{2}} A\left[\Sigma_{t}\right]\right|_{t=0}=\frac{1}{2} \int_{C} \omega\left|\partial_{\bar{z}} y^{i}\right|^{2}
$$

which appears as the bosonic term in (2.45). This formula indicates that holomorphic curves are always area-minimizing in $X$, and only holomorphic deformations of a holomorphic curve can preserve its area.

Finally, to make contact with the finite-dimensional model, we can evaluate the partition function $Z(C)$ of a $D 1$-brane wrapped on $C$ exactly as we evaluated the contribution to the finite-dimensional integral from an isolated, non-degenerate zero of $s$ in (2.13). We find that

$$
\begin{aligned}
Z(C) & =\int_{\mathcal{M}} \operatorname{Pfaff}\left(\bar{\partial}_{E(-1)}\right) d \mu e^{-S}, \\
& =\frac{\operatorname{Pfaff}\left(\bar{\partial}_{E(-1)}\right)(C)}{\operatorname{det}\left(\delta^{2} \Psi / \delta y^{j} \delta y^{i}\right)(C)} .
\end{aligned}
$$

Here $d \mu=\mathcal{D} y^{i} \mathcal{D} y^{\bar{i}} \epsilon^{\dot{\alpha} \dot{\beta}} \mathcal{D} \psi_{\dot{\beta}}^{\bar{j}} \mathcal{D} \psi_{\dot{\alpha}, j}$ is the naive path-integral measure, and the Pfaffian factor produced by the left-moving bundle fermions is directly analogous to the section $g$, since both are required for the path-integral measure to be well-defined. Recalling from (2.41) that $\delta^{2} \Psi / \delta y^{j} \delta y^{i}$ represents the $\bar{\partial}$ operator acting on sections of $N=\mathcal{O}(-1) \oplus \mathcal{O}(-1)$, we see that $Z(C)$ indeed agrees with the summand in the expression (11.3). 


\section{A Residue Theorem for the Heterotic String}

We now extend our investigation of residues in Section 2 to the heterotic string 8 itself. Our goal is to prove a residue theorem, precisely analogous to the theorems we derived in Section 2, for the vanishing of world-sheet instanton contributions to $W$. A very useful tool in our analysis is the twisted version of the heterotic world-sheet theory, as it is the twisted theory that directly generalizes the finite-dimensional model we introduced in Section 2. Thus, we begin this section with a short reminder of what it means to twist [2832] the heterotic world-sheet theory, and we explain how this theory is related to the finite-dimensional model of Section 2.

\subsection{Preliminary Remarks on Twisting}

The twisted heterotic world-sheet theory is simply a version of the physical (untwisted) heterotic theory in which the right-moving world-sheet fermions are assigned unconventional spins. To describe the twisting, we first recall that the world-sheet theory contains complex bosons $\phi^{i}$ and $\phi^{\bar{i}} \equiv \overline{\phi^{i}}$ which describe sigma model maps $\Phi: \Sigma \rightarrow X$ from the world-sheet $\Sigma$ to a Kähler target space $X$. In the physical theory, the superpartners of $\phi^{i}$ and $\phi^{\bar{i}}$ are right-moving fermions $\psi^{i}$ and $\psi^{\bar{i}}$, which transform as sections of the bundles $\bar{K}^{\frac{1}{2}} \otimes \Phi^{*}(T X)$ and $\bar{K}^{\frac{1}{2}} \otimes \Phi^{*}(\overline{T X})$ respectively. Here, $T X$ denotes the holomorphic tangent bundle of $X$, and $\bar{K}$ denotes the anti-canonical bundle of $\Sigma$. The anti-canonical bundle can be explicitly described as the line-bundle of $(0,1)$ forms on $\Sigma$, and from this description we see that $\bar{K}^{\frac{1}{2}}$ is a right-moving spin-bundle on $\Sigma$. Then in the twisted theory, we simply take $\psi^{i}$ to transform as a section of $\bar{K} \otimes \Phi^{*}(T X)$ and $\psi^{\bar{i}}$ to transform as a section of $\Phi^{*}(\overline{T X})$.

One way to interpret the twist is that we shift the right-moving world-sheet stress tensor $T_{\overline{z z}}$ by

$$
T_{\overline{z z}} \rightarrow \widetilde{T}_{\overline{z z}}=T_{\overline{z z}}+\frac{1}{2} \partial_{\bar{z}} j_{\bar{z}},
$$

where $j_{\bar{z}}$ is the world-sheet $U(1)$ current present in the right-moving $N=2$ algebra. Upon twisting, one of the two right-moving world-sheet supersymmetry generators becomes a nilpotent scalar $Q$, which we interpret as a $B R S T$-operator on the world-sheet. The decoupling of $Q$-trivial states from the correlation functions of $Q$-invariant operators then

8 Because the left-moving world-sheet fermions play only an auxiliary role in our analysis, we will not need to distinguish between the $E_{8} \times E_{8}$ and $\operatorname{Spin}(32) / \mathbf{Z}_{2}$ heterotic strings. 
greatly simplifies the twisted theory. In particular, though the twisted heterotic theory is not topological, all correlation functions of $Q$-invariant operators in the twisted theory vary holomorphically on the world-sheet, because the twisted stress-tensor $\widetilde{T}_{\overline{z z}}$ is $Q$-trivial.

We now explain how the general framework of Section 2 applies to the twisted worldsheet theory. Instead of performing an integral over a finite-dimensional complex manifold $M$, we now perform a path-integral over the infinite-dimensional complex manifold $\mathcal{M}$ which is the space of all sigma model maps $\Phi: \Sigma \rightarrow X$. The world-sheet bosons $\phi^{i}$ themselves provide local holomorphic coordinates on $\mathcal{M}$ and play the same role as the holomorphic coordinates $z^{i}$ on $M$. In addition, the fermions $\psi^{\bar{i}}$, as sections of $\Phi^{*}(\overline{T M})$, are coordinates on $\overline{T \mathcal{M}}$ and correspond to the anti-commuting coordinates $\theta^{\bar{i}}$ in Section 2. Finally, we interpret the fermion $\$ \frac{9}{z}$, which transform as sections of $\bar{K} \otimes \Phi^{*}(T X)$, as anti-commuting coordinates on a holomorphic bundle $\mathcal{V}$ over $\mathcal{M}$, so that these fermions play the same role as the fermionic coordinates $\chi^{\alpha}$ on $V$ in Section 2. In particular, on world-sheets for which $\bar{K}$ is trivial, we can identify the bundle $\mathcal{V}$ on $\mathcal{M}$ as the holomorphic tangent bundle $T \mathcal{M}$.

Under $Q$, the world-sheet fields transform as

$$
\begin{array}{ll}
\delta \phi^{i}=0, & \delta \phi^{\bar{i}}=\psi^{\bar{i}}, \\
\delta \psi_{\bar{z}}^{i}=\partial_{\bar{z}} \phi^{i}, & \delta \psi^{\bar{i}}=0 .
\end{array}
$$

Comparing (3.2) to (2.1), we see that the action of $Q$ is precisely analogous to the supersymmetry transformation in the finite-dimensional model. Further, we see that $\partial_{\bar{z}} \phi^{i}$ is the holomorphic section of $\mathcal{V}$ corresponding to the section $s$ of $V$ in Section 2.

The sigma model action for the world-sheet fields can now be written as

$$
S=\int_{\Sigma} d^{2} z \delta\left(g_{\bar{i} i} \partial_{z} \phi^{\bar{i}} \psi \frac{i}{z}\right)+\cdots,
$$

where $g_{\bar{i} i}$ is the Kähler metric on $X$. The $Q$-trivial expression above is a direct generalization of the action (2.4) which we considered in Section 2. Just as the finite-dimensional integral localizes on the set where $s=0$, so the twisted path-integral localizes on sigma model maps satisfying $\partial_{\bar{z}} \phi^{i}=0$. Such maps, being holomorphic, are either constant or represent world-sheet instantons.

9 We have slightly changed notation $\psi^{i} \rightarrow \psi \frac{i}{z}$ to remind ourselves that $\psi \frac{i}{z}$ now transforms as a $(0,1)$ form on $\Sigma$. 
The "..." appearing in $S$ represents the additional terms in the sigma model action which are not $Q$-trivial (but of course are $Q$-closed). More precisely, these additional terms arise either from a purely topological expression which is the integral of the complexified Kähler class of $X$ over $\Sigma$ or from the kinetic terms of the left-moving bundle fermions.

Both of these sorts of terms admit an easy interpretation in light of the results of Section 2. First, if we restrict the world-sheet path-integral to the sector describing maps whose images lie in a fixed homology class of $X$, the topological term in $S$ is constant and can be ignored. Second, if we also only consider world-sheet correlation functions which do not involving the left-moving bundle fermions, then at least for isolated world-sheet instantons, the only role of the bundle fermions is to produce the Pfaffian factor that appears in the Introduction. As we have already observed in the contex of the D1-brane, like the section $g$ in the finite-dimensional model, this Pfaffian factor can be interpreted as defining a suitable measure for the path-integral over the modes of $\phi^{i}, \phi^{\bar{i}}, \psi \frac{i}{z}$, and $\psi^{\bar{i}}$.

Finally we remark that, although the physical and twisted theories are generally very different, some quantities in the physical theory can be computed using the twisted theory. In particular, as long as $K$ is the trivial bundle on $\Sigma$, correlation functions computed on $\Sigma$ in the twisted theory agree with those computed on $\Sigma$ in the physical theory. For instance, if $\Sigma$ is a cylinder with Ramond sector ground-states at each end, then correlation functions on $\Sigma$ compute the Yukawa couplings arising from the superpotential $W$ in the low-energy effective theory. In this fashion we can use the twisted theory to probe for a background $W$.

\subsection{The Half-Linear Heterotic String}

Our proof of the residue theorem in Section 2 only relies upon the fact that the integral $Z$ is invariant under a nilpotent supersymmetry and the fact that the space $M$ over which we integrate is compact. We wish to generalize this residue theorem to apply to worldsheet correlators in the twisted heterotic theory, so we must consider models for which both of these crucial facts hold. Since the action of the BRST-operator $Q$ on the world-sheet naturally generalizes the supersymmetry transformation of Section 2, the first fact holds for an arbitrary $(0,2)$ compactification. However, as regards the second fact, the space $\mathcal{M}$ of sigma model maps is certainly not compact, and so to generalize the finite-dimensional residue theorem from Section 2 to a vanishing result for $W$ of the form (1.3), we must look for heterotic models with some special sort of compactness. 
The vanishing result of [4] naturally suggests that we start by considering the linear sigma models. Indeed, the compactness of the moduli spaces of $X$ and $E$ is an essential ingredient in the analysis of [⿴囗十 .

Moreover, the linear sigma models possess another sort of compactness not present in an arbitrary heterotic compactification. As discussed extensively in [33] and [34, in compactifications for which continuous families of world-sheet instantons exist, the instanton moduli spaces of the linear sigma model provide natural compactifications of the instanton moduli spaces of the corresponding nonlinear sigma model. The compactness of these instanton moduli spaces turns out to be the essential ingredient in our proof of a residue theorem for the heterotic string.

However, we do not really have to consider the linear sigma models themselves to exploit the fact that the instanton moduli spaces of the corresponding nonlinear sigma models have natural compactifications. We find it technically simpler, in fact, to discuss a class of half-linear heterotic models. These models are like the linear models in that $X$ is a complete-intersection Calabi-Yau in a compact toric variety $Y$. Unlike the linear models, the gauge bundle $E$ on $X$ is any bundle which satisfies the usual consistency conditions on $X$ and also pulls back from a bundle on $Y$. Thus $E$ must generally be described in a nonlinear fashion.

So in the remainder of this section, we first introduce the half-linear models and demonstrate that the finite-dimensional residue theorems of Section 2 naturally generalize to formulae of the form (1.3). We then return to the linear sigma models themselves and give a direct proof of the vanishing of instanton contributions to $W$. For concreteness, we shall throughout this section consider only the case that $X$ is the quintic hypersurface in $Y=\mathbf{C P}^{4}$.

\section{Half-linear fields}

We start by specifying the field content of the half-linear model. The world-sheet bosons and the right-moving world-sheet fermions are the usual fields which describe twisted nonlinear sigma model maps $\Phi: \Sigma \rightarrow Y$. For the case $Y=\mathbf{C P}^{4}$, the model has four complex bosons $\phi^{i}$ and $\phi^{\bar{i}}$ which represent local holomorphic and anti-holomorphic coordinates on $Y$ (as opposed to the global homogeneous coordinates on $Y$ that would appear in the corresponding linear sigma model). Since the half-linear model is twisted, the right-moving supersymmetry associates to the bosons $\phi^{i}$ and $\phi^{\bar{i}}$ corresponding fermions $\psi \frac{i}{z}$ and $\psi^{\bar{i}}$, transforming on $\Sigma$ as sections of $\bar{K} \otimes \Phi^{*}(T Y)$ and $\Phi^{*}(\overline{T Y})$ respectively. 
As for the left-moving sector of the world-sheet, the bundle $E$ on $Y$ is represented in the usual nonlinear fashion by a set of thirty-two left-moving fermions $\lambda^{a}$ coupled to the pull back of $E$ to $\Sigma$. We assume that $E$ satisfies the standard topological conditions for anomaly-cancellation and stability on $X$. Thus, $E$ satisfies $p_{1}(E) / 2=c_{2}(T X)$ (and, if the structure group of $E$ reduces to a subgroup with $U(1)$ factors, there are restrictions on the corresponding first Chern classes).

However, the field content of the half-linear model, as it stands, cannot be correct. As in the linear sigma model, to localize the half-linear model from $Y$ onto $X$, we must introduce a potential $J\left(\phi^{i}\right)$ on the world-sheet. Geometrically, $J$ transforms as a holomorphic section of the line-bundle $\mathcal{O}(5)$ on $Y$. Supersymmetry requires that $J$ couple to the right-moving fermions as well as the bosons, but we currently have no way to couple $J$ to these fermions.

A more fundamental problem is that, although we choose the bundle $E$ so as to cancel sigma model anomalies on $X$, the half-linear model on $Y$ is currently anomalous as $p_{1}(E) / 2 \neq c_{2}(T Y)$.

We can elegantly fix both of these problems by adding a pair of left-moving fermions to the model. These fermions, which we denote by $\chi_{z}$ and $\bar{\chi}$, transform on the world-sheet as sections of $K \otimes \Phi^{*}(\mathcal{O}(-5))$ and $\Phi^{*}(\mathcal{O}(5))$. Thus we can directly include the required Yukawa terms for $J$ in the model.

As for the anomalies, since $\chi_{z}$ and $\bar{\chi}$ are also "twisted" in the sense of having nonstandard world-sheet spins, they cancel the excess left-moving central charge from the new boson. Also, upon adding $\chi_{z}$ and $\bar{\chi}$ to the left-moving sector of the model, we cancel the sigma model anomalies, since near $X$, the adjunction formula implies that $T Y$ splits as a smooth bundle into the sum $T X \oplus \mathcal{O}(5)$. Explicitly, relative to the model on $X$, the half-linear model on $Y$ has an additional pair of twisted, right-moving fermions which arise from the directions in the normal bundle $\mathcal{O}(5)$ to $X$ in $Y$. These fermions transform as sections of $\bar{K} \otimes \Phi^{*}(\mathcal{O}(5))$ and $\Phi^{*}(\mathcal{O}(-5))$. Since $\chi_{z}$ and $\bar{\chi}$ transform as the complex conjugates of these two fermions, they cancel the corresponding anomalies. 


\section{Half-linear supersymmetry}

In the half-linear model, the action of the scalar supercharge $Q$ slightly generalizes (3.2), due to the transformations of the left-moving fermions $\chi_{z}$ and $\bar{\chi}-$ the other leftmoving fermions are invariant. So $Q$ acts as

$$
\begin{array}{ll}
\delta \phi^{i}=0, & \delta \phi^{\bar{i}}=\psi^{\bar{i}}, \\
\delta \psi_{\bar{z}}^{i}=\partial_{\bar{z}} \phi^{i}, & \delta \psi^{\bar{i}}=0, \\
\delta \bar{\chi}=J\left(\phi^{i}\right), & \\
\delta \chi_{z}=0 . &
\end{array}
$$

As we have mentioned, $J$ is locally a quintic polynomial in the holomorphic coordinates $\phi^{i}$ and globally a holomorphic section of $\mathcal{O}(5)$ on $Y$. Of course, $J$ represents the data needed to determine $X$ as a hypersurface in $Y$.

We see from the action of $Q$ that the fermions $\psi_{\bar{z}}^{i}, \bar{\chi}$, and $\chi_{z}$ in the half-linear model can all be identified as the analogues of the fermions $\chi^{\alpha}$ in the finite-dimensional model. In this basis, $s=\left(\partial_{\bar{z}} \phi^{i}, J\left(\phi^{i}\right), 0\right)$. So, if we construct an action for the half-linear model analogous to (2.4) in the finite-dimensional model, the half-linear model will localize on sigma model maps $\Phi$ satisfying

$$
\partial_{\bar{z}} \phi^{i}=J\left(\phi^{i}\right)=0 .
$$

The first condition requires that $\Phi$ be holomorphic, and the second condition requires that the image of $\Phi$ lie in the subset $J=0$ of $Y$, which can be identified with $X$. So the half-linear model localizes on world-sheet instantons in $X$.

\section{The half-linear action}

To complete our description of the half-linear model, we must finally specify its worldsheet action $S$.

First, in complete analogy to the action of the finite-dimensional integral, $S$ includes the terms

$$
\begin{aligned}
S_{0} & =t \int_{\Sigma} d^{2} z \delta\left(g_{\bar{i} i} \partial_{z} \phi^{\bar{i}} \psi \bar{z}+\bar{J} \bar{\chi}\right) \\
& =t \int_{\Sigma} d^{2} z\left(g_{\bar{i} i} \partial_{z} \phi^{\bar{i}} \partial_{\bar{z}} \phi^{i}+g_{\bar{i} i} D_{z} \psi^{\bar{i}} \psi \bar{z}+\bar{J} J+\psi^{\bar{i}} D_{\bar{i}} \bar{J} \bar{\chi}\right) .
\end{aligned}
$$

Here $t$ is a coupling parameter as in Section 2, and $g_{\bar{i} i}$ is a Kähler metric on $Y$. Because $S_{0}$ is $Q$-exact, quantities which we compute in the half-linear model are unchanged under deformations of $t, g_{\bar{i} i}$, and $\bar{J}$. We also observe parenthetically that, since the expression 
$\bar{J} \bar{\chi}$ transforms as a smooth section of the trivial bundle on $Y$, we do not actually need to specify a hermitian bundle metric on $\mathcal{O}(5)$ to make sense of this expression.

The action $S_{E}$ for the left-moving fermions $\lambda^{a}$ which describe $E$ is the standard action, which we record for completeness below,

$$
S_{E}=\int_{\Sigma} d^{2} z\left(\lambda_{a} D \bar{z}^{a} \lambda^{a}+F_{\bar{i} i b}^{a} \lambda_{a} \lambda^{b} \psi^{\bar{i}} \psi_{\bar{z}}^{i}\right)
$$

where

$$
\lambda_{a} D_{\bar{z}} \lambda^{a}=\lambda_{a} \partial_{\bar{z}} \lambda^{a}+\lambda_{a} \partial_{\bar{z}} \phi^{i} A_{i b}^{a} \lambda^{b} .
$$

In the above, $A_{i b}^{a}$ is a holomorphic connection on $E$, having components only of type $(1,0)$ on $Y$, and $F_{\bar{i} i b}{ }^{a}$ is the curvature of this connection. Since $S_{E}$ is the usual action for the left-moving bundle fermions, and since $Q$ acts in the usual way (3.2) on the fields appearing in $S_{E}$, this action is clearly $Q$-invariant.

A more nontrivial fact is that we can also write a $Q$-invariant action for the fermions $\chi_{z}$ and $\bar{\chi}$. Abstractly, the presence of $\chi_{z}$ and $\bar{\chi}$ in the half-linear model implies that we are dealing with the over-determined case $\operatorname{dim} M<\operatorname{rank} V$ discussed in Section 2.3. So we must add some $Q$-invariant observable $\mathcal{O}$ involving $\chi_{z}$ and $\bar{\chi}$ to the action if we wish to compute something nontrivial in the half-linear model.

Physically, this $Q$-invariant observable $\mathcal{O}$ must introduce a kinetic term $D \bar{z} \bar{\chi} \chi_{z}$ for $\chi_{z}$ and $\bar{\chi}$. To find a $Q$-invariant extension of this kinetic term, we follow the philosophy of Section 2.3 and attempt to solve $\delta \mathcal{O}=0$ perturbatively. We begin by noting that the expression $\mathcal{O}^{(0)}$,

$$
\begin{aligned}
\mathcal{O}^{(0)} & =D_{\bar{z}} \bar{\chi} \chi_{z}-\psi \psi_{\bar{z}}^{i} D_{i} J \chi_{z} \\
& =\left(\partial_{\bar{z}}+\partial_{\bar{z}} \phi^{i} A_{i}\right) \bar{\chi} \chi_{z}-\psi \frac{i}{z}\left(\partial_{i}+A_{i}\right) J \chi_{z},
\end{aligned}
$$

is trivially invariant under the variations of $\bar{\chi}$ and $\psi \frac{i}{z}$. That is, in analogy to the finitedimensional model, $\iota_{s} \mathcal{O}^{(0)}=0$.

In the expression for $\mathcal{O}^{(0)}$ above, we have introduced the canonical holomorphic connection $A_{i}$ on the line-bundle $\mathcal{O}(5)$ on $Y$. Because $A_{i}$ depends on $\phi^{\bar{i}}$ as well as $\phi^{i}$, we have that

$$
\delta \mathcal{O}^{(0)}=\bar{\partial} \mathcal{O}^{(0)} \neq 0
$$

Rather,

$$
\bar{\partial} \mathcal{O}^{(0)}=F_{\bar{i} i} \psi^{\bar{i}} \partial_{\bar{z}} \phi^{i} \bar{\chi} \chi_{z}-F_{\bar{i} i} \psi^{\bar{i}} \psi_{\bar{z}}^{i} J \chi_{z}
$$


where $F_{\bar{i} i}$ is the curvature of $A_{i}$. However, introducing $\mathcal{O}^{(1)}$,

$$
\mathcal{O}^{(1)}=F_{\bar{i} i} \psi^{\bar{i}} \psi_{\bar{z}}^{i} \bar{\chi} \chi_{z}
$$

we easily see that

$$
\bar{\partial} \mathcal{O}^{(0)}+\iota_{s} \mathcal{O}^{(1)}=0
$$

Because $A_{i}$ is a holomorphic connection on $\mathcal{O}(5)$, the curvature satisfies $\bar{\partial} F_{\bar{i} i}=0$, so that $\bar{\partial} \mathcal{O}^{(1)}=0$. Consequently, $\mathcal{O}=\mathcal{O}^{(0)}+\mathcal{O}^{(1)}$ is $Q$-invariant (but not $Q$-trivial).

Thus, we can add kinetic terms for $\chi_{z}$ and $\bar{\chi}$ to the action $S$ by including

$$
\begin{aligned}
S_{\chi} & =\int_{\Sigma} d^{2} z \mathcal{O}=\int_{\Sigma} d^{2} z\left(\mathcal{O}^{(0)}+\mathcal{O}^{(1)}\right) \\
& =\int_{\Sigma} d^{2} z\left(D \bar{z} \bar{\chi} \chi_{z}-\psi \frac{i}{z} D_{i} J \chi_{z}+F_{\bar{i} i} \psi^{\bar{i}} \psi \frac{i}{z} \bar{\chi} \chi_{z}\right)
\end{aligned}
$$

Finally, we include in $S$ the purely topological term which describes the action of the world-sheet instanton itself,

$$
S_{t o p}=\int_{\Sigma} \Phi^{*}\left(\omega_{\mathbf{C}}\right),
$$

where $\omega_{\mathbf{C}}$ is the complexified Kähler class of $Y$. This term simply reproduces the exponential factor in (1.2), but we include it for completeness.

Thus, the action for the half-linear model is

$$
S=S_{0}+S_{E}+S_{\chi}+S_{t o p}
$$

\subsection{A Half-Linear Residue Theorem}

We now show in the half-linear model that world-sheet instanton contributions to the superpotential $W$ vanish by a residue theorem precisely analogous to the finite-dimensional residue theorem of Section 2.

Before we discuss a residue theorem for the half-linear model, though, we must first demonstrate the general fact that the half-linear model on $Y$ is equivalent to the usual twisted non-linear sigma model on $X$. Only then does the residue theorem for the halflinear model imply the vanishing of the instanton contributions to $W$ in the non-linear sigma model.

Relative to the non-linear model on $X$, the half-linear model on $Y$ possesses additional world-sheet degrees of freedom described by the left-moving fermions $\chi_{z}, \bar{\chi}$, and the 
complex boson and associated right-moving fermions describing fluctuations of the worldsheet normal to $X$ in $Y$. We will denote these normal fields simply by $\phi, \bar{\phi}, \psi_{\bar{z}}$, and $\bar{\psi}$, suppressing indices associated to the tangent bundle $T Y$.

The additional world-sheet fields present in the half-linear model on $Y$ relative to the non-linear model on $X$ are all massive due to the terms in the action involving $J$ and $\bar{J}$. For instance, the normal bosons $\phi$ and $\bar{\phi}$ gain a mass from the $\bar{J} J$ term that appears in the $Q$-trivial action $S_{0}$,

$$
t \int_{\Sigma} d^{2} z \delta(\bar{J} \bar{\chi})=t \int_{\Sigma} d^{2} z\left(\bar{J} J+\psi^{\bar{i}} D_{\bar{i}} \bar{J} \bar{\chi}\right) .
$$

Similarly, the fermions $\psi_{\bar{z}}, \bar{\psi}, \chi_{z}$, and $\bar{\chi}$ all gain masses from the $\overline{D J}$ term in (3.17) and the conjugate term appearing in $S_{\chi}$ in (3.14).

The mass terms for $\phi, \bar{\phi}, \psi_{\bar{z}}, \bar{\psi}, \chi_{z}$, and $\bar{\chi}$ thus appear in the half-linear action as

$$
\int_{\Sigma} d^{2} z\left(\bar{\phi} \overline{D J} D J \phi+\bar{\psi} \overline{D J} \bar{\chi}-\psi_{\bar{z}} D J \chi_{z}\right)
$$

where $D J$ is the (holomorphic) normal derivative of $J$ along $X$, and we have absorbed the coupling $t$ in (3.17) into $\bar{J}$. Because we assume that $X$ is a non-singular quintic hypersurface, $D J$ is everywhere non-vanishing on $X$ and consequently transforms in the trivial line-bundle on $X$. Also, because $J$ is holomorphic and vanishes on $X$, the $\bar{\partial}$ operator of $X$ acts on $D J$ as $\bar{\partial} D J=[\bar{\partial}, D] J=0$, so that $D J$ is holomorphic on $X$. As such, once we choose a non-vanishing holomorphic section of the trivial bundle on $X$, a choice which we must make in defining the fermionic measure of the path-integral, we can regard $D J$ as merely a constant mass parameter for the normal modes.

As we have already remarked, since $\bar{J}$ only appears in the half-linear model through the $Q$-trivial terms in (3.17), the half-linear model is invariant under deformations of $\bar{J}$. Scaling $\bar{J}$ by a large constant, the massive world-volume fields in (3.18) all acquire arbitrarily large masses. 10 As such, we can integrate out these massive world-sheet fields at one-loop with arbitrary precision. From (3.18), we see that the one-loop contributions from the massive modes of $\phi, \bar{\phi}, \psi_{\bar{z}}, \bar{\psi}, \chi_{z}$, and $\bar{\chi}$ all cancel but for a finite, anomalous factor associated to the index of the $\bar{\partial}$ operator acting on the pull back of the normal bundle $N$ to the

10 Looking back at (3.18), the $\overline{D J} D J$ term is a mass for scalars of order $\sqrt{|\bar{J}|}$. The $\bar{\psi} \overline{D J} \bar{\chi}$ term is part of a fermion mass matrix $\left(\begin{array}{cc}0 & D J \\ D J & 0\end{array}\right)$ where the upper right entry comes from the $\psi D J \chi$ term; it again leads to masses of order $\sqrt{|\bar{J}|}$. 
world-sheet. This one-loop contribution can be absorbed into a renormalization of the string coupling constant and the Kähler class of $X$ and is not relevant for the vanishing argument. Finally, upon integrating out the massive fields, we set them to zero in the halflinear action and in all observables, so that the half-linear model on $Y$ clearly localizes to the non-linear model on $X$.

For completeness, we give in this paragraph a brief description of the renormalization. Massive modes with nonzero momentum cancel in the path integral, so the renormalization comes from the constant modes. The constant modes contribute a factor

$$
\frac{1}{(\overline{D J} D J)^{n_{1}}} \overline{D J}^{n_{2}} D J^{n_{3}}
$$

where $n_{1}$ formally denotes the number of modes of $\phi, n_{2}$ the number of modes of $\bar{\chi}$, and $n_{3}$ the number of modes of $\chi_{z}$. Since $\phi$ and $\bar{\chi}$ both transform in the pull back $\Phi^{*}(N)$ of the normal bundle $N=\mathcal{O}(5)$ to the world-sheet, $n_{1}$ equals $n_{2}$. However, $\chi_{z}$ transforms in the bundle $K \otimes \Phi^{*}\left(N^{*}\right)$, and thus the difference $n_{1}-n_{3}$ is equal to the index of the $\bar{\partial}$ operator on the world-sheet acting on the bundle $\Phi^{*}(N)$. If the world-sheet is a Riemann surface $\Sigma$ of genus $g$ and $\Phi$ is a map of degree $d$ into $X$, then the index theorem (or simply the Riemann-Roch theorem) implies that $n_{1}-n_{3}=5 d+1-g$. Thus, in this situation the one-loop contribution of the massive modes is a factor $(1 / D J)^{(5 d+1-g)}$. In the non-linear model on $X$, this one-loop contribution can be written as

$$
\left(\frac{1}{D J}\right)^{(5 d+1-g)}=\exp \left[-\frac{1}{2 \pi} \int_{\Sigma} \log (D J)\left(5 \Phi^{*}(\omega)+\frac{1}{2} R\right)\right]
$$

where $\Phi^{*}(\omega)$ is the pull back of the Kähler class from $X$, which we assume is normalized to satisfy $\int_{\Sigma} \Phi^{*}(\omega)=2 \pi d$, and $R$ is the world-sheet curvature, which satisfies $\int_{\Sigma} R=$ $4 \pi(1-g)$. The expression in (3.20) manifestly represents the renormalization of the Kähler class of $X$ and the string coupling constant upon integrating out the massive modes.

Having shown that the half-linear model on $Y$ is equivalent to the non-linear sigma model on $X$, we now establish a residue theorem for the half-linear model which implies the vanishing of world-sheet instanton contributions to the superpotential $W$.

The half-linear model is a closer cousin to the usual world-sheet CFT description of the heterotic string than to the dual D1-brane description which we explored in Section 2.4. As such, in neither the world-sheet CFT nor the half-linear model can we compute $W$ directly. Rather, because of the presence of three left- and right-moving fermion zero-modes arising from fluctuations tangent to the world-sheet, we must indirectly probe for $W$ by computing 
a cubic correlator of vertex operators on the world-sheet. In the terminology of Section 2.3, the half-linear model describes the over-determined case $\operatorname{rank} \mathcal{V}>\operatorname{dim} \mathcal{M}$, due to the presence of the fermions $\chi_{z}$ and $\bar{\chi}$ in the model, but the section $s=\left(\partial_{\bar{z}} \phi^{i}, J\left(\phi^{i}\right), 0\right)$ still vanishes over a locus on $\mathcal{M}$ of complex dimension three, due to the $S L(2, \mathbf{C})$ action on the world-sheet. So we must insert a suitable observable $\mathcal{O}$, the cubic correlator of vertex operators, to compute something non-trivial.

The easiest way to probe for $W$ is to compute the correlator $\langle R R R\rangle$, where $R$ is the vertex operator for the (unique) Kähler modulus of $Y$. Explicitly,

$$
R=\omega_{i i} \partial_{z} \phi^{i} \psi^{\bar{i}}
$$

where $\omega_{\bar{i} i}$ is a harmonic representative of the Kähler class of $Y$, implying that $R$ is $Q^{-}$ invariant. Since the half-linear model arises from a sigma model on $Y$ (and only restricts to $X$ when $\bar{J}$ is large), we must consider operators such as $R$ which are actually defined on $Y$. Note as claimed that each of the three right-moving fermion zero-modes from $S L(2, \mathbf{C})$ can be soaked up with the fermion $\psi^{\bar{i}}$ that appears in $R$.

Of course, the Kähler class of $Y$ determines by restriction the Kähler class of $X$ and thus the radius of the compactification. The only dependence of $W$ on this Kähler modulus is through the exponential factor $\exp \left(-\int_{\Sigma} \Phi^{*}(\omega)\right)$ arising from the classical action of the instanton itself. If we let $\mathcal{R}$ be the $\mathcal{N}=1$ chiral field in the low-energy effective theory associated to the Kähler modulus, then the correlator $\langle R R R\rangle$ computes the third derivative $\partial_{\mathcal{R}}^{3} W$ of $W$ with respect to $\mathcal{R}$. Thus, given the simple exponential dependence of $W$ on $\mathcal{R}$, the vanishing of $W$ is equivalent to the vanishing of the correlator $\langle R R R\rangle$.

In the case of the finite-dimensional model in Section 2, we deduced a residue theorem by taking $t=0$. Although we have already interpreted the half-linear model as being formally analogous to the finite-dimensional model, unlike the case of the finite-dimensional model, we cannot simply take $t=0$ in the half-linear model to deduce that $\langle R R R\rangle$ vanishes. Clearly with no exponential suppression of the fluctuating modes in the half-linear model, the half-linear path-integral ceases to be defined.

However, in localizing the half-linear model on $Y$ to the non-linear model on $X$, we have already used the fact that the $B R S T$-invariance of the half-linear model implies that the model is formally independent of $\bar{J}$ as well as $t$. So rather than taking $t=0$, we consider taking $\bar{J}=0$ instead. 
When $\bar{J}=0$, the half-linear model no longer localizes on instantons contained in $X$. Instead, after integrating out at weak coupling all fluctuating modes of the fields, the half-linear model localizes onto the moduli space of instantons in $Y$.

If we restrict attention to a given instanton sector of degree $d$ holomorphic maps $\Phi$ from $\Sigma=\mathbf{C P}^{1}$ to $Y$, then the moduli space of these instantons has a natural compactification to $\mathbf{C P}^{5 d+4}$. Because of this compactness, the half-linear path-integral over each instanton sector can be defined even when $\bar{J}=0$. Thus, the correlator $\langle R R R\rangle$ can be computed either at large $\bar{J}$, where it is proportional to $W$ as computed in the non-linear model on $X$, or it can be computed at $\bar{J}=0$, where we will easily see that it vanishes order by order in $d$. Morally speaking, the vanishing of the instanton contribution to the superpotential follows by applying the residue theorem of section 2 to the compact manifold $\mathbf{C P}^{5 d+4}$. Rather than invoking this theorem (which could lead one to worry about singularities in $\mathbf{C P}^{5 d+4}$ ), we will imitate its proof and just look at what happens at $\bar{J}=0$.

We now review in detail how $\mathbf{C P}^{5 d+4}$ arises as a compactification of the moduli space of degree $d$ instantons in $Y$, following [5, 33, 34]. In fact, even though we focus here on the case $Y=\mathbf{C P}^{4}$, the existence of such a compactification generalizes whenever $Y$ is a compact toric variety, as already applied in [33,34].

We first introduce homogeneous coordinates $\left[\Phi^{0}: \cdots: \Phi^{4}\right]$ on $Y$ and homogeneous coordinates $[U: V]$ on $\Sigma$. In terms of the homogeneous coordinates, any degree $d$ holomorphic map $\Phi: \Sigma \rightarrow Y$ is specified by a set of homogeneous, degree $d$ polynomials $\left\{p^{0}(U, V), \ldots, p^{4}(U, V)\right\}$,

$$
\begin{aligned}
\Phi^{0}= & p^{0}(U, V)=a_{0}^{0} U^{d}+a_{1}^{0} U^{d-1} V+\cdots+a_{d}^{0} V^{d}, \\
& \vdots \\
\Phi^{4}= & p^{4}(U, V)=a_{0}^{4} U^{d}+a_{1}^{4} U^{d-1} V+\cdots+a_{d}^{4} V^{d} .
\end{aligned}
$$

Each polynomial $p^{i}$ is determined by its $d+1$ coefficients $\left(a_{0}^{i}, \ldots, a_{d}^{i}\right)$, and the space of polynomials $\left\{p^{0}, \ldots, p^{4}\right\}$ can be parametrized by these coefficients as $\mathbf{C}^{5(d+1)}$. Since the coordinates $\left[\Phi^{0}: \cdots: \Phi^{4}\right]$ are merely homogeneous coordinates on $Y$, defined only up to scaling, an overall scaling of $\left\{p^{0}, \ldots, p^{4}\right\}$ does not affect the map $\Phi$. Subtracting from $\mathbf{C}^{5(d+1)}$ the point at the origin which does not describe an actual map into $Y$ and then taking the quotient by the overall scaling, we find the projective space $\mathbf{C P}^{5 d+4}$.

The only subtlety in this example is that, as just observed, $\Phi^{0}=\cdots=\Phi^{4}=0$ does not correspond to any point in $Y$, so that the moduli space of instantons of degree $d$ on $Y$ 
is actually the subset of the parameter space $\mathbf{C P}^{5 d+4}$ for which the polynomials $p^{0}, \ldots, p^{4}$ have no common zeroes on $\Sigma$. The polynomials which do have at least one common zero appear as an algebraic locus of codimension four in $\mathbf{C P}^{5 d+4}$, since we must tune one complex parameter in any four of $p^{0}, \ldots, p^{4}$ to reach this locus. Thus, the moduli space of "true" instantons in $Y$ is a complicated but nonetheless dense, open subset of $\mathbf{C P}^{5 d+4}$. In particular, $\mathbf{C P}^{5 d+4}$ gives a natural compactification of the true moduli space.

We now consider evaluating the correlator $\langle R R R\rangle$ in the half-linear model with $\bar{J}=0$. In this case, if we consider the contribution to the correlator from the topological sector of degree $d$ world-sheet maps, we must integrate over the moduli space of degree $d$ instantons in $Y$ described globally above.

This integral over the instanton moduli space is actually a supersymmetric integral just as in Section 2, since both the world-sheet bosons and fermions possess zero-modes when $\bar{J}=0$. As our global discussion above implies, the bosons $\phi^{i}, \phi^{\bar{i}}$, and their superpartners $\psi^{\bar{i}}$ all have $5 d+4$ zero-modes. Of these $5 d+4$ zero-modes, three zero-modes arise from the $S L(2, C)$ action on $\mathbf{C P}^{1}$ and are immediately soaked up by the cubic correlator. The other $5 d+1$ zero-modes represent the non-trivial holomorphic deformations of degree $d$ rational curves in $Y$. The left-moving fermion $\bar{\chi}$ also has $5 d+1$ zero-modes, which arise from holomorphic sections of the bundle $\Phi^{*}(\mathcal{O}(5))=\mathcal{O}(5 d)$. Neither the right-moving fermions $\psi \frac{i}{z}$ nor the left-moving fermion $\chi_{z}$ have any zero-modes in the instanton background.

When $\bar{J}$ is non-vanishing, these $5 d+1$ interesting modes of $\phi^{i}, \phi^{\bar{i}}, \psi^{\bar{i}}$, and $\bar{\chi}$ enter the half-linear model action through the $Q$-trivial terms involving $\bar{J}$ in (3.17) and through the four-fermion interactions in (3.7) and (3.14). In the weak coupling limit $t \rightarrow \infty$, the four-fermion interactions are irrelevant, since they always involve the fermions $\psi \frac{i}{z}$ which have no zero-modes. So the only way to absorb the zero-modes of $\psi^{\bar{i}}$ and $\bar{\chi}$ is through the quadratic mass terms that arise from $\bar{J}$.

In fact, if we consider integrating out all of the fluctuating modes at weak coupling, to reduce the half-linear path-integral to a finite-dimensional supersymmetric integral over these $5 d+1$ modes, then the $Q$-trivial terms involving $\bar{J}$ in (3.17) implicitly represent the same finite-dimensional action (2.5) which we considered in Section 2. In this case, the modes of $\psi^{\bar{i}}$ represent the fermionic coordinates $\theta^{\bar{i}}$, the modes of $\bar{\chi}$ represent the bundle fermions $\chi^{\alpha}$, and $J$ implicitly determines a holomorphic section $s$ of a rank $5 d+1$ bundle over the moduli space of instantons in $Y$ which vanishes precisely over those instantons contained in $X$. 
Just as in Section 2 , once we set $\bar{J}$ to zero, then the $5 d+1$ fermion zero-modes of $\psi^{\bar{i}}$ and $\bar{\chi}$ cannot be absorbed when computing the correlator $\langle R R R\rangle$. Hence, $\langle R R R\rangle$ vanishes order by order for each sector of degree $d$ maps. Finally, since our vanishing result follows exactly as the residue theorem in Section 2, we naturally interpret it as a residue theorem for instanton contributions to $W$.

\subsection{Extension to the Linear Sigma Model}

Just as in the finite-dimensional case, the argument for the vanishing of the instanton contributions to $W$ in the half-linear model relies only upon the right-moving world-sheet supersymmetries and suitable compactness. These ingredients are also present in the $(0,2)$ linear sigma models themselves, so we should also be able to give a similar, direct argument for the vanishing of instanton contributions to $W$ in these models. The reason for doing so is that the linear sigma model version of the argument applies to a somewhat different class of models - bundles constructed in a simple way from polynomials, but which do not necessarily extend over $Y=\mathbf{C P}^{4}$.

We now present just such an argument. Although the gist of the vanishing argument for the linear sigma model is exactly the same as for the half-linear model, we must present the details of the argument in a slightly different way, since the specifics of the linear model and the half-linear model are very different. Nonetheless, the fact that the general argument does extend from the half-linear to the linear model, despite the obvious differences between these world-sheet theories, indicates that this argument is robust.

As in the previous section, we once more focus on the case that $X$ is a quintic hypersurface in $Y=\mathbf{C P}^{4}$. However, we now assume that the bundle $E$ on $X$ is a deformation of the holomorphic tangent bundle $T X$, which corresponds in the linear sigma model to a deformation away from the locus of theories with $(2,2)$ world-sheet supersymmetry. Since neither $T X$ nor $E$ pulls back (in any obvious way) from any bundle on $Y$, the compactifications cannot necessarily be described by the half-linear model.

\section{Background}

We must recall a few facts about the $(0,2)$ linear sigma model which describes heterotic compactification on $X$ with gauge bundle $E$. Useful background can be found in [4], [5], and [6]. We will be rather brief in our description of the linear sigma model, both because this material is well-known and also because the vanishing argument which we present does not rely on many details of the model. 
We first recall the field content 11 for this model. On the $(2,2)$ locus itself, the linear sigma model which describes a quintic $X$ in $\mathbf{C P}^{4}$ is a two-dimensional $U(1)$ gauge theory with five chiral superfields $S^{i}, i=1, \ldots, 5$, of charge +1 and one chiral superfield $P$ of charge -5 .

Once this model is deformed away from the $(2,2)$ locus, the $(2,2)$ gauge multiplet decomposes into a $(0,2)$ gauge multiplet and a neutral $(0,2)$ chiral multiplet. Similarly, each $(2,2)$ chiral multiplet decomposes into a $(0,2)$ chiral multiplet and a $(0,2)$ Fermi multiplet. We denote again by $S^{i}$ and $P$ the corresponding $(0,2)$ chiral superfields, with components $\left(s^{i}, \psi_{+}^{i}\right)$ and $\left(p, \psi_{+}^{0}\right)$, and by $\Psi_{-}^{i}$ and $\Psi^{0}$ the associated Fermi superfields, with components $\psi_{-}^{i}$ and $\psi_{-}^{0}$.

The action of the $(0,2)$ model contains many interactions, but the only interactions relevant to our vanishing argument arise from the $(0,2)$ superpotential. Recall that these interactions can be written as integrals over half of $(0,2)$ superspace, in the form

$$
S_{J}=\frac{1}{\sqrt{2}} \int_{\Sigma} d \theta^{+}\left(\Psi_{-}^{i} J_{i}+\Psi_{-}^{0} J_{0}\right)+\text { h.c. }
$$

In general, $J_{i}$ and $J_{0}$ are holomorphic functions of the chiral fields $S^{i}$ and $P$. More specifically, $J_{i}$ and $J_{0}$ take the form

$$
\begin{aligned}
J_{i} & =P\left(\frac{\partial F}{\partial S^{i}}+F_{i}\right), \quad i=1, \ldots, 5 \\
J_{0} & =F
\end{aligned}
$$

where $F=F\left(S^{i}\right)$ is a quintic polynomial in the $S^{i}$ which determines $X$ as a hypersurface in $Y$, and the $F_{i}$ are quartic polynomials in the $S^{i}$ that are assumed to satisfy $S^{i} F_{i}=0$ and which determine $E$ as a deformation of $T X$.

In terms of the component fields, the $(0,2)$ superpotential (3.23) leads to a bosonic potential $U$,

$$
U=\sum_{i=0}^{5}\left|J_{i}\right|^{2}
$$

and Yukawa interactions of the form

$$
\psi_{-}^{i} \psi_{+}^{j} \frac{\partial J_{i}}{\partial S^{j}}+\psi_{-}^{i} \psi_{+}^{0} \frac{\partial J_{i}}{\partial P}+\text { h.c. }
$$

11 We ignore the decoupled current algebra degrees of freedom which represent the unbroken space-time gauge group. 
The superpotential (3.23) also preserves a right-moving $U(1) \mathcal{R}$-symmetry, under which the lowest components of $S^{i}$ and $\Psi_{-}^{i}$ carry charge $+\frac{1}{5}$, and the lowest components of $P$ and $\Psi_{-}^{0}$ are neutral.

\section{The vanishing theorem}

The first step in our vanishing argument is to twist the $(0,2)$ linear sigma model so that the supersymmetry generator usually denoted $\bar{Q}_{+}$becomes a scalar, exactly as described in [4]. Under this twisting, the world-sheet spin of each field is shifted by $-\frac{1}{2} J_{R}+\frac{1}{10} Q$, where $J_{R}$ is the $\mathcal{R}$-symmetry generator and $Q$ is the gauge-symmetry generator. Since the gauge current corresponding to $Q$ is of the form $\left\{\bar{Q}_{+}, \ldots\right\}$, the fact that $\frac{1}{10} Q$ appears in the twist is irrelevant and is merely for convenience, so that upon twisting all fields have integral or half-integral world-sheet spins.

Upon twisting the model, the spins of the bosons $s^{i}$ are unaffected, but the boson $p$ now has spin $-\frac{1}{2}$ and transforms as a section of $K^{\frac{1}{2}} \otimes \mathcal{L}^{-5}$ on $\Sigma=\mathbf{C P}^{1}$. Here $K$ is the canonical bundle on $\Sigma$ as earlier, and $\mathcal{L}=\mathcal{O}(d)$ is the line-bundle on $\Sigma$ associated to a degree $d$ instanton configuration in the gauge field. Also, just as in the half-twisted model, the fermions $\psi_{+}^{i}$ and $\psi_{+}^{\bar{i}}$, for $i=1, \ldots, 5$, now have spins +1 and 0 and transform as sections of $\bar{K} \otimes \mathcal{L}$ and $\overline{\mathcal{L}}$. Finally, the left-moving fermions $\psi_{-}^{i}$ and $\psi_{-}^{\bar{i}}$ are unaffected by the twisting and transform as sections of $K^{\frac{1}{2}} \otimes \mathcal{L}$ and $K^{\frac{1}{2}} \otimes \overline{\mathcal{L}}$.

To proceed with the argument, we must compute the linear sigma model correlator analogous to $\langle R R R\rangle$ in the half-linear model. As explained in [甘], the linear sigma model representative of the vertex operator $R$ describing deformations of the Kähler class of $Y$ (hence also $X$ ) is $\lambda_{-}$, the left-moving gaugino. This fact can be motivated by observing that, since the Kähler class of $Y$ is represented in the linear model by a Fayet-Iliopolous $D$-term, the linear sigma model representative for $R$ must come from the $(0,2)$ gauge multiplet. The supersymmetry and $\mathcal{R}$-symmetry then determine this representative to be $\lambda_{-}$. So we must compute the instanton contributions to $\left\langle\lambda_{-} \lambda_{-} \lambda_{-}\right\rangle$in the linear sigma model.

As in the half-linear model, the twisted linear model is formally invariant under deformations of $\bar{J}_{i}, i=0, \ldots, 5$, so we consider taking $\bar{J}_{i}=0$. At first glance, one might worry that this deformation would be singular in the linear model, since at least in the untwisted theory, the boson $p$ has an unbounded zero-mode which only receives a mass from the potential term $U$ in (3.25). However, because $p$ has spin $-\frac{1}{2}$ in the twisted theory, this dangerous zero-mode is not present. This observation was also central to the vanishing 
argument of [1], so we certainly expect it to play a role in our argument as well. Thus, we can compute $\left\langle\lambda_{-} \lambda_{-} \lambda_{-}\right\rangle$in the theory with $\bar{J}_{i}=0$, provided we perform the twist.

In the half-linear model, once we performed the analogous deformation by taking $\bar{J}=0$, we easily saw that the correlator $\langle R R R\rangle$ vanished due to the presence of excess fermion zero-modes which could no longer be absorbed through world-sheet interactions. We will now argue that the correlator $\left\langle\lambda_{-} \lambda_{-} \lambda_{-}\right\rangle$vanishes when $\bar{J}_{i}=0$ in the linear sigma model, again due to excess fermion zero-modes.

The relevant zero-modes arise from the fermions $\psi_{+}^{\bar{i}}$ and $\psi_{-}^{i}$, for $i=1, \ldots, 5$. In the background of a degree $d$ instanton, each fermion $\psi_{+}^{\bar{i}}$ has $d+1$ zero-modes, and each fermion $\psi_{-}^{i}$ has $d$ zero-modes (and the conjugate partners of these fermions have no zero-modes).

To show that these fermion zero-modes cannot be absorbed in computing the correlator $\left\langle\lambda_{-} \lambda_{-} \lambda_{-}\right\rangle$with $\bar{J}_{i}=0$, we first make a few general remarks about the computation of $\left\langle\lambda_{-} \lambda_{-} \lambda_{-}\right\rangle$even when the $\bar{J}_{i}$ are not assumed to vanish. First, since all kinetic terms in the linear model are $\bar{Q}_{+}$-trivial, we can by a field rescaling assume that the couplings appearing in $J_{i}$ and $\bar{J}_{i}$ are arbitrarily small. Hence we can compute $\left\langle\lambda_{-} \lambda_{-} \lambda_{-}\right\rangle$perturbatively in $J_{i}$ and $\bar{J}_{i}$.

As a special case of our vanishing result, we now observe that $\left\langle\lambda_{-} \lambda_{-} \lambda_{-}\right\rangle$trivially vanishes when $J_{i}=\bar{J}_{i}=0$. In this case, the model with no superpotential describes, instead of $X$, the total space of the line-bundle $\mathcal{O}(-5)$ over $\mathbf{C P}^{4}$. As such, the model possesses a classical global symmetry which rotates the fiber of this space leaving fixed the base. Under this symmetry, the superfields $S^{i}$ and $\Psi_{-}^{i}$, for $i=1, \ldots, 5$, transform with charge +1 while all other fields are uncharged. In particular, the gaugino $\lambda_{-}$is uncharged, which distinguishes this global symmetry from the $\mathcal{R}$-symmetry.

The fermion zero-modes we discussed above are relevant precisely because they cause this classical symmetry to be anomalous. Due to these zero-modes, regardless of the degree $d$, the path-integral measure transforms with net charge +5 under this symmetry. This anomaly immediately implies that $\left\langle\lambda_{-} \lambda_{-} \lambda_{-}\right\rangle$vanishes in the theory with no superpotential. For instance, computing $\left\langle\lambda_{-} \lambda_{-} \lambda_{-}\right\rangle$perturbatively at weak coupling, all interactions respect the classical symmetry and so there is no way to absorb the fermion zero-modes by pulling down fermion interaction terms from the action. This fact is why the detailed structure of the linear model is largely irrelevant for our argument.

We now consider the general case that $J_{i}$ and $\bar{J}_{i}$ are non-zero. Since the superpotential breaks the classical symmetry we used above, the fermion Yukawa terms involving $J_{i}$ and $\bar{J}_{i}$ in $(\overline{3.26})$ are candidates to soak up the zero-modes of $\psi_{+}^{\bar{i}}$ and $\psi_{-}^{i}$ above. However, 
whatever interaction terms we bring down from the action to soak up the fermion zeromodes, the anomaly implies that these terms must carry net charge -5 to cancel the charge of the measure. We now observe from (3.26) that the interactions involving $J_{i}$ all carry charge +5 and those involving $\bar{J}_{i}$ carry charge -5 .

Thus, when $\bar{J}_{i}$ vanishes, the zero-modes of the fermions $\psi_{+}^{\bar{i}}$ and $\psi_{-}^{i}$ cannot be absorbed, since perturbation theory in the $J_{i}$ can only bring down interactions of positive charge. This observation merely reflects the fact that the twisted fermions $\psi_{+}^{\bar{i}}$, which give rise to the anomaly, appear in the Yukawa couplings involving $\bar{J}_{i}$, not $J_{i}$, in (3.26). Thus $\left\langle\lambda_{-} \lambda_{-} \lambda_{-}\right\rangle$vanishes in an arbitrary degree $d$ instanton background, and instantons in the linear sigma model do not contribute to the space-time superpotential.

Of course, when $\bar{J}_{i}$ is non-zero, the linear model sums over individual instantons in $X$, and the contribution of each instanton should generically be non-zero. Our argument is consistent with this fact, since insertions of the Yukawa couplings involving both $J_{i}$ and $\bar{J}_{i}$ can carry the proper charge to absorb the zero-modes.

This vanishing argument is at its heart very similar to the vanishing argument of [4] that we reviewed in the Introduction. A key fact there is that $W$ transforms as a section of a line-bundle of strictly negative curvature on the moduli space of the low-energy effective theory. Now in the context of the present argument, the complex coefficients which define the quintic polynomial $F$ and the quartic polynomials $F_{i}$, and thus appear as couplings in the $J_{i}$, can be considered as projective coordinates on the moduli space of complex structures of $X$ and $E$.

Our perturbative argument above can be rephrased as a selection rule for the dependence of the correlator $\left\langle\lambda_{-} \lambda_{-} \lambda_{-}\right\rangle$on these coefficients. This selection rule follows from formally assigning the complex coefficients appearing in $F$ and $F_{i}$ charge -5 under the anomalous symmetry, so that formally the $J_{i}$ are uncharged. The anomaly implies that the correlator $\left\langle\lambda_{-} \lambda_{-} \lambda_{-}\right\rangle$, as a function of these coefficients, transforms homogeneously with charge +5 . As a result, the selection rule implies that $\left\langle\lambda_{-} \lambda_{-} \lambda_{-}\right\rangle$(and hence $W$ ) must transform as a section of a line-bundle of strictly negative curvature over the complex structure moduli space which these coefficients parametrize. In this language, our vanishing theorem follows simply because, when $\bar{J}_{i}$ vanishes, a perturbative calculation of $\left\langle\lambda_{-} \lambda_{-} \lambda_{-}\right\rangle$in terms of the $J_{i}$ can only produce a polynomial in the complex coefficients, which has negative charge under the anomalous symmetry and does not have the required pole on the complex structure moduli space. 


\section{Families of Membrane Instantons}

The vanishing result which we derived for world-sheet instanton contributions to the superpotential is a manifestation of the rigidity inherent in holomorphic objects. As an interesting contrast to this result, we now consider how M-theory membranes which wrap a continuous family of supersymmetric three-cycles in a manifold $X$ of $G_{2}$ holonomy contribute to the superpotential. The approach which we take here is very similar to our discussion of D1-brane contributions to the superpotential at the end of Section 2. We note that the superpotential contribution from an isolated membrane in $X$ has already been thoroughly discussed in [15].

Just as in the case of a D1-brane which wraps a holomorphic curve, the worldvolume theory on a membrane which wraps a supersymmetric three-cycle on $X$ is naturally twisted. Unlike the case of the D1-brane though, in the case of a supersymmetric membrane, the sector of the worldvolume theory describing fluctuations in $X$ is topological, as opposed to holomorphic, in character. This fact could hardly be otherwise, since $X$ is not a complex manifold, but it represents a key distinction between $D 1$-brane and membrane instantons.

Thus, if $\mathcal{C}$ represents a continuous family of supersymmetric membrane configurations within the space $\mathcal{M}$ of all membrane configurations in $X$, then the contribution to the superpotential from the family $\mathcal{C}$ only depends upon topological data associated to $\mathcal{C}$. Our main result here is to show that the contribution of the family $\mathcal{C}$ to the superpotential is proportional to the Euler character $\chi(\mathcal{C})$ of $\mathcal{C}$.

Our analysis ignores singularities. We suspect that it remain valid even if some of the membrane instantons parametrized by $\mathcal{C}$ are singular, as long as $\mathcal{C}$ itself is smooth. A simple example in which this is the case is that $X$ is a $T^{3}$-fibered $G_{2}$ manifold, in which case $\mathcal{C}$ is a $\mathrm{K} 3$ surface, of Euler character 24.

\section{The membrane worldvolume theory}

Just as in the Introduction, the most elegant way to determine the superpotential contribution from a membrane instanton (or a family of such instantons) is to compute the partition function of the membrane worldvolume theory. The structure of this theory is largely determined by supersymmetry. More specifically, it is determined by the requirement that only supersymmetric membrane configurations contribute to the partition function. So we begin by recalling a few facts about supersymmetric three-cycles in $X$.

To describe which three-cycles in $X$ are supersymmetric, we first recall that $X$, as a manifold of $G_{2}$ holonomy, possesses a canonical, covariantly constant three-form $\phi$. Then, 
as emphasized generally in [35], the supersymmetric three-cycles are those which are calibrated by $\phi$ and hence are of minimal volume within each homology class. That is, if $\Sigma$ is a supersymmetric three-cycle, then the calibration condition states that on $\Sigma$,

$$
\left.\phi\right|_{\Sigma}=\left.\operatorname{vol}\right|_{\Sigma}
$$

where vol $=\frac{1}{7} \phi \wedge \star \phi$ is the volume form associated to the metric on $X$.

Just as for supersymmetric D1-brane configurations, the supersymmetric membrane configurations in $X$ can be characterized as the critical points of a superpotential $\Psi$ on M. $\Psi$ is defined in a manner precisely analogous to the superpotential for D1-brane configurations in a Calabi-Yau threefold. Thus, we define $\Psi(\Sigma)$ for any three-cycle $\Sigma$ by

$$
\Psi(\Sigma)-\Psi\left(\Sigma_{0}\right)=\frac{1}{12} \int_{B} \star \phi
$$

Here $\star \phi$ is the four-form on $X$ dual to $\phi, \Sigma_{0}$ is a fixed representative in the homology class of $\Sigma$, and $B$ is a four-cycle bounding $\Sigma-\Sigma_{0}$. Again, $\Psi(\Sigma)$ is defined only up to an additive constant, depending on the choices of $\Sigma_{0}$ and $B$.

But again, the fact that $\Psi$ is only defined up to an additive constant does not concern us, as this constant does not affect the location of the critical points, for which $\delta \Psi=0$. In terms of local coordinates $x^{i}, i=1, \ldots, 7$, on $X$,

$$
\delta \Psi(\Sigma)=\frac{1}{3} \int_{\Sigma} \star \phi_{i j k l} \delta x^{i} d x^{j} \wedge d x^{k} \wedge d x^{l}
$$

Thus, $\delta \Psi(\Sigma)=0$ when $\star \phi_{i j k l} d x^{j} \wedge d x^{k} \wedge d x^{l}=0$ on $\Sigma$. As observed in [36], this condition is equivalent to the condition (4.1) that $\Sigma$ be calibrated by $\phi$. So the critical points of $\Psi$ correspond to supersymmetric three-cycles in $X$.

Thus, $\delta \Psi$ is a one-form on the space $\mathcal{M}$ of arbitrary membrane configurations in $X$ which vanishes precisely over the supersymmetric configurations. So in this sense, $\delta \Psi$ plays much the same role as the section $s$ we introduced in Section 2, and we expect the action of the worldvolume theory on a supersymmetric membrane to be expressed in terms of $\delta \Psi$, much as the action (2.5) is expressed in terms of $s$.

Unlike $s$, though, $\delta \Psi$ is not holomorphic, and the space $\mathcal{M}$ of membrane configurations is not even complex, even on-shell. As a result, the supersymmetry algebra on the membrane worldvolume takes a form slightly different from the supersymmetry (2.1) considered in Section 2. 
We focus on the sector of the worldvolume theory which describes fluctuations of the membrane in $X$. As explicitly demonstrated in [15], this sector is automatically twisted when the membrane wraps a supersymmetric cycle $\Sigma$. Normal fluctuations of the membrane in $X$ are described on the worldvolume by four real bosons $y^{i}, i=1, \ldots, 4$, taking values in the (real) normal bundle $N$ of $\Sigma$ in $X$. Associated to these four bosons are four fermions $\psi_{\dot{\alpha}}^{i}$ also taking values in $N$ and transforming as right-moving Weyl fermions in $\mathbf{R}^{4}$, as indicated by the $\dot{\alpha}$ index.

The worldvolume theory on the supersymmetric membrane then possesses two scalar supercharges $\bar{Q}_{\dot{\alpha}}$. The action of these supercharges on the worldvolume fields can be neatly summarized by introducing $(0 \mid 2)$ superfields $Y^{i}$, where

$$
Y^{i}=y^{i}+\theta^{\dot{\alpha}} \psi_{\dot{\alpha}}^{i}+\frac{1}{2} \epsilon_{\dot{\alpha} \dot{\beta}} \theta^{\dot{\alpha}} \theta^{\dot{\beta}} F^{i} .
$$

In defining the superfield $Y^{i}$, we have introduced an auxiliary boson $F^{i}$ taking values in $N$. Even though the membrane worldvolume is three-dimensional, the appropriate superspace is only the (0|2) superspace because, just as for the D1-brane, we regard the bosonic fields $y^{i}$ as being an infinite set of tangential coordinates to the membrane configuration space $\mathcal{M}$ at the point corresponding to a given supersymmetric membrane configuration.

In the $(0 \mid 2)$ superspace, the action of the supercharges $\bar{Q}_{\dot{\alpha}}$ is exceedingly simple. Namely, the supercharges $\bar{Q}_{\dot{\alpha}}$ act as the fermionic derivatives $\partial_{\dot{\alpha}}$, corresponding to the component transformations

$$
\delta_{\dot{\alpha}} y^{i}=\psi_{\dot{\alpha}}^{i}, \quad \delta_{\dot{\alpha}} \psi_{\dot{\beta}}^{i}=\epsilon_{\dot{\alpha} \dot{\beta}} F^{i}, \quad \delta_{\dot{\alpha}} F^{i}=0 .
$$

We note that $\left\{\bar{Q}_{\dot{\alpha}}, \bar{Q}_{\dot{\beta}}\right\}$ trivially vanishes.

The supersymmetry algebra, along with the requirement that the membrane partition function localize on configurations for which $\delta \Psi=0$, determines the form of the worldvolume action on a supersymmetric membrane. As for the D1-brane, this action is really the leading order action for fluctuations around a supersymmetric configuration but given the topological nature of the membrane worldvolume theory, the leading order action certainly suffices to determine the partition function.

When written in terms of the $(0 \mid 2)$ superspace, the membrane worldvolume action thus appears as

$$
\begin{aligned}
S & =\int_{\Sigma} d^{2} \theta \phi\left(\frac{1}{2} g_{i j}(Y) \epsilon^{\dot{\alpha} \dot{\beta}} \partial_{\dot{\beta}} Y^{i} \partial_{\dot{\alpha}} Y^{j}+\Psi(Y)\right) \\
& =\int_{\Sigma} \phi\left(\frac{1}{2} g^{i j} \frac{\delta \Psi}{\delta y^{i}} \frac{\delta \Psi}{\delta y^{j}}+2 \frac{D^{2} \Psi}{D y^{i} D y^{j}}\left(\psi^{i} \psi^{j}\right)+R_{i k j l}\left(\psi^{i} \psi^{j}\right)\left(\psi^{k} \psi^{l}\right)\right) .
\end{aligned}
$$


In this expression, $g_{i j}$ is the metric on $X, R_{i k j l}$ is the curvature, and the canonical threeform $\phi$ appears simply to represent the volume-form on the supersymmetric three-cycle $\Sigma$. We also note from (4.3) that $\delta \Psi / \delta y^{i}$ is actually a three-form on $\Sigma$, and so we have implicitly used the induced metric to dualize $\delta \Psi / \delta y^{i}$ to a scalar above. Finally, we have used the shorthand $\left(\psi^{i} \psi^{j}\right)$ to indicate the $S U(2)$ singlet combination $\frac{1}{2} \epsilon^{\dot{\alpha} \dot{\beta}} \psi_{\dot{\beta}}^{i} \psi_{\dot{\alpha}}^{j}$, and in passing to the second line of (4.6) we integrated out the auxiliary bosons $F^{i}$.

The membrane worldvolume action (4.6) has a very familiar look. Formally, we can interpret this action as the reduction to $0+0$ dimensions of the standard supersymmetric quantum mechanics [37] on the membrane configuration space $\mathcal{M}$, with Morse function $\Psi$. As is well known, the partition function of supersymmetric quantum mechanics on a finite-dimensional Riemannian manifold $M$ computes the Euler class $\chi(M)$ of $M$. Thus, our claim that the membrane partition function is proportional to the Euler class $\chi(\mathcal{C})$ of the family $\mathcal{C}$ follows almost immediately now, though we still discuss this result in detail below.

We can also compare the form of the membrane worldvolume theory to the form of the D1-brane worldvolume theory (or more generally to the holomorphic models we considered in Section 2). Upon integrating out the auxiliary bosons $F^{i}$, the supersymmetries on the membrane worldvolume act as

$$
\begin{aligned}
\delta_{\dot{\alpha}} y^{i} & =\psi_{\dot{\alpha}}^{i}, \\
\delta_{\dot{\alpha}} \psi_{\dot{\beta}}^{i} & =-\Gamma_{j k}^{i} \psi_{\dot{\alpha}}^{j} \psi_{\dot{\beta}}^{k}+\frac{1}{2} \epsilon_{\dot{\alpha} \dot{\beta}} g^{i j} \frac{\delta \Psi}{\delta y^{j}} .
\end{aligned}
$$

In the above, $\Gamma_{j k}^{i}$ is the usual torsion-free affine connection associated to the metric $g_{i j}$ on $X$; this connection must appear so that the fermions $\psi_{\dot{\alpha}}^{i}$ transform covariantly under reparametrizations of the $y^{i}$. Comparing the supersymmetries (4.7) and action (4.6) of the membrane worldvolume theory to the general supersymmetry (2.1) and action (2.5) from Section 2, we see that the membrane worldvolume theory is just a real, $N=2$ version of the holomorphic models relevant for world-sheet instantons which we considered earlier. Clearly the one-form $\delta \Psi$ on $\mathcal{M}$ plays exactly the same role as the holomorphic section $s$ on the complex manifold $M$, and from (4.6) we see that at weak coupling the membrane partition function localizes on the zeroes of $\delta \Psi$. We also note that the $N=$ 2 supersymmetry present in the membrane worldvolume theory determines a canonical choice for the measure of the membrane partition function, as all bosons are paired by supersymmetry with all fermions in (4.5). So there is no analogue here of the section $g$ which was necessary to define a measure for the holomorphic models. 


\section{The membrane partition function}

Our simple description of the membrane worldvolume theory allows us to easily evaluate the membrane partition function, even in the degenerate case that the membranes wrap a continuous family of supersymmetric three-cycles in $X$.

We first observe that, because $\bar{Q}_{\dot{\alpha}}=\partial_{\dot{\alpha}}$, the worldvolume action (4.6) is evidently $\bar{Q}_{\dot{\alpha}^{-}}$ trivial. As a result, the membrane partition function $Z$ is clearly topological in character. In particular, $Z$ is unchanged if we multiply $\phi \rightarrow t \phi$, so that taking $t$ to be large we can evaluate $Z$ at weak coupling. Furthermore, $Z$ is unchanged under deformations of the metric $g_{j k}$ and even the one-form $\delta \Psi$. This latter observation is in clear contrast to the holomorphic models in Section 2, which were unchanged under deformations of $\bar{s}$ but certainly depended upon $s$.

Thus, we suppose that $X$ contains a continuous family of supersymmetric three-cycles. Then the vanishing locus of $\delta \Psi$ on $\mathcal{M}$ contains a component $\mathcal{C}$ of positive dimension representing this continuous family. To evaluate $Z$ for membranes which wrap three-cycles in $\mathcal{C}$, we simply make a generic deformation of $\delta \Psi$, which is small in the sense that $\delta \Psi$ still grows sufficiently fast away from $\mathcal{C}$ so that $Z$ is defined. Under such a deformation, we lift the degeneracy of $\delta \Psi$, which now has a finite set of isolated zeroes on $\mathcal{C}$.

At weak coupling, we can directly evaluate the contribution to $Z$ from each nondegenerate zero of $\delta \Psi$ as a one-loop integral over the fluctuating bosons and fermions. Generally speaking, if $P$ is such a zero, then the contribution to $Z$ from $P$ takes the form

$$
Z_{P}=Z(\mathcal{N}) \cdot Z(\mathcal{C})_{P},
$$

where $Z(\mathcal{N})$ represents the one-loop integral over modes normal to $\mathcal{C}$, and $Z(\mathcal{C})_{P}$ represents the one-loop integral over the finite number of modes tangent to $\mathcal{C}$ at $P$. Because of the topological invariance of $Z$, the factor $Z(\mathcal{N})$ in (4.8) does not depend on $P$, so that

$$
Z=Z(\mathcal{N}) \cdot \sum_{P} Z(\mathcal{C})_{P} .
$$

Clearly the second factor in (4.9) captures the interesting dependence of the superpotential on $\mathcal{C}$. In the Gaussian approximation, we can express the contribution $Z(\mathcal{C})_{P}$ from each point $P$ as

$$
Z(\mathcal{C})_{P}=\frac{\operatorname{det}_{\mathcal{C}}\left(\partial_{i} \partial_{j} \Psi\right)(P)}{\left|\operatorname{det}_{\mathcal{C}}\left(\partial_{i} \partial_{j} \Psi\right)(P)\right|}= \pm 1
$$


where the subscript $\mathcal{C}$ indicates that the determinants are only evaluated over the modes tangent to $\mathcal{C}$. Geometrically, we recognize the expression (4.10) as the index of the vector field $\nabla \Psi$ (projected onto $T \mathcal{C}$ ) at the point $P$, where it vanishes. Thus,

$$
\sum_{P} Z(\mathcal{C})_{P}=\chi(\mathcal{C})
$$

and $Z$ is proportional to the Euler character $\chi(\mathcal{C})$ of $\mathcal{C}$ as claimed. We could also derive this result, without explicitly deforming $\delta \Psi$ to lift its degeneracy, by using the four-fermion interaction in (4.6) to absorb the fermion zero-modes tangent to $\mathcal{C}$, producing the ChernWeil representation of the Euler character.

Finally, we remark that the factor $Z(\mathcal{N})$, studied in [15] for the case of an isolated membrane instanton, is simply the formal generalization of (4.10) from the phase of a determinant on the tangential directions of $\mathcal{C}$ to the normal directions. $Z(\mathcal{N})$ can thus be expressed as the sign of the Dirac operator acting on the membrane worldvolume spinors multiplied by a factor coming from the $C$-field.

\section{Acknowledgements}

The work of C.B. is supported by a National Science Foundation Graduate Fellowship and under NSF Grant PHY-9802484. The work of E.W. is supported in part by NSF Grant PHY-0070928.

Any opinions, findings, and conclusions or recommendations expressed in this material

are those of the authors and do not necessarily reflect the views of the National Science Foundation. 


\section{References}

[1] M. Dine, N. Seiberg, X. G. Wen, and E. Witten, "Nonperturbative Effects on the String Worldsheet, I, II," Nucl. Phys. B278 (1986) 769-789, Nucl. Phys. B289 (1987) 319-363.

[2] J. Distler, "Resurrecting (2, 0) Compactifications," Phys. Lett. B188 (1987) 431-436.

[3] J. Distler and B. Greene, "Aspects of $(2,0)$ String Compactifications, Nucl. Phys. B304 (1988) 1-62.

[4] E. Silverstein and E. Witten, "Criteria For Conformal Invariance of $(0,2)$ Models," Nucl. Phys. B444 (1995) 161-190, hep-th/9503212.

[5] E. Witten, "Phases of $\mathrm{N}=2$ Theories in Two-Dimensions," Nucl. Phys. B403 (1993) 159-222, hep-th/9301042.

[6] J. Distler, "Notes on $(0,2)$ Superconformal Field Theories," hep-th/9502012.

[7] K. Becker, M. Becker, and A. Strominger, "Fivebranes, Membranes, and NonPerturbative String Theory," Nucl. Phys. B456 (1995) 130-152, hep-th/9507158.

[8] E. Witten, "World-Sheet Corrections via D-Instantons," JHEP 0002 (2000) 030, hep-th/9907041.

[9] J. Polchinski and E. Witten, "Evidence for Heterotic-Type I String Duality," Nucl. Phys. B460 525-540, hep-th/9510169.

[10] E. I. Buchbinder, R. Donagi, and B. A. Ovrut, "Superpotentials for Vector Bundle Moduli," hep-th/0205190.

[11] E. I. Buchbinder, R. Donagi, and B. A. Ovrut, "Vector Bundle Moduli Superpotentials in Heterotic Superstrings and M-Theory," JHEP 0207 (2002) 066, hep-th/0206203.

[12] P. Griffiths and J. Harris, Principles of Algebraic Geometry, John Wiley and Sons, Inc., New York, 1978.

[13] A. K. Tsikh, Multidimensional Residues and Their Applications, Trans. E. J. F. Primrose, American Mathematical Society, Providence, R. I., 1992.

[14] A. Basu and S. Sethi, "World-sheet Stability of $(0,2)$ Linear Sigma Models," hep-th/ 0303066 .

[15] J. A. Harvey and G. Moore, "Superpotentials and Membrane Instantons," hep-th/ 9907026 .

[16] J. B. Carrell and D. I. Lieberman, "Holomorphic Vector Fields and Kaehler Manifolds," Invent. Math. 21 (1973) 303-309.

[17] J. B. Carrell and D. I. Lieberman, "Vector Fields and Chern Numbers," Math. Ann. 225 (1977) 263-273.

[18] K. Liu, "Holomorphic Equivariant Cohomology," Math. Ann. 303 (1995) 125-148.

[19] E. Witten, "Topological Quantum Field Theory," Commun. Math. Phys. 117 (1988) $353-386$. 
[20] S. Cordes, G. Moore, and S. Ramgoolam, "Lectures on 2D Yang-Mills Theory, Equivariant Cohomology, and Topological Field Theories," Nucl. Phys. Proc. Suppl. 41 (1995) 184-244, hep-th/9411210.

[21] R. Bott and L. W. Tu, Differential Forms in Algebraic Topology, Springer, New York, 1982.

[22] M. Bershadsky, V. Sadov, and C. Vafa, "D-Branes and Topological Field Theories," Nucl Phys. B463 (1996) 420-434, hep-th/9511222.

[23] S. K. Donaldson and R. P. Thomas, "Gauge Theory in Higher Dimensions," in The Geometric Universe: Science, Geometry, and the Work of Roger Penrose, Ed. S. A. Huggett et. al., Oxford University Press, Oxford, 1998.

[24] E. Witten, "Branes and the Dynamics of QCD," Nucl. Phys. B507 (1997) 658-690, hep-th/9706109.

[25] N. C. Leung, "Topological Quantum Field Theory for Calabi-Yau Threefolds and $G_{2}$ Manifolds," math.DG/0208124.

[26] J. Simons, "Minimal Varieties of Riemannian Manifolds," Ann. of Math. 88 (1968) $62-105$.

[27] H. B. Lawson, Jr., Minimal Varieties in Real and Complex Geometry, Séminaire de Mathématiques Supérieures Université de Montréal, 1974.

[28] E. Witten, "Topological Sigma Models," Commun. Math. Phys. 118 (1988) 411-449.

[29] E. Witten, "Mirror Manifolds and Topological Field Theory," in Essays on Mirror Manifolds, ed. S.-T. Yau, International Press (1992) 120-160, hep-th/9112056.

[30] T. Eguchi and S. K. Yang, "N=2 Superconformal Models as Topological Field Theories," Mod. Phys. Lett. A5 (1990) 1693-1701.

[31] I. Antoniadis, E. Gava, K. S. Narain, and T. R. Taylor, "Topological Amplitudes in String Theory," Nucl. Phys. B413 (1994) 162-184, hep-th/9307158.

[32] I. Antoniadis, E. Gava, K. S. Narain, and T. R. Taylor, "Topological Amplitudes in Heterotic Superstring Theory,"Nucl. Phys. B476 (1996) 133-174, hep-th/9604077.

[33] D. R. Morrison and M. R. Plesser, "Summing the Instantons: Quantum Cohomology and Mirror Symmetry in Toric Varieties," Nucl. Phys. B440 (1995) 279-354, hep-th/ 9412236 .

[34] A. Losev, N. Nekrasov, and S. Shatashvili, "The Freckled Instantons," hep-th/ 9908204.

[35] S. Gukov, "Solitons, Superpotentials, and Calibrations," Nucl. Phys. B574 (2000) 169-188, hep-th/9911011.

[36] R. Harvey and H. B. Lawson, "Calibrated Geometries," Acta Math. 148 (1982) 47157.

[37] E. Witten, "Supersymmetry and Morse Theory," J. Differential Geometry 17 (1982) 661-692. 